\title{
Adaptive observers for TS fuzzy systems with unknown polynomial inputs
}

\author{
Zs. Lendek ${ }^{a, *}$, J. Lauber $^{\mathrm{b}}$, T. M. Guerra ${ }^{\mathrm{b}}$, R. Babuška ${ }^{\mathrm{a}}$, B. De Schutter ${ }^{\mathrm{a}, \mathrm{c}}$ \\ ${ }^{a}$ Delft Center for Systems and Control, Delft University of Technology \\ Mekelweg 2, 2628 CD Delft, The Netherlands \\ ${ }^{b}$ Université de Valenciennes, BP 311, Le Mont Houy, 59313 Valenciennes Cedex 9 \\ ${ }^{c}$ Marine and Transport Technology Department, Delft University of Technology, The \\ Netherlands
}

\begin{abstract}
A large class of nonlinear systems can be well approximated by TakagiSugeno (TS) fuzzy models, with linear or affine consequents. However, in practical applications, the process under consideration may be affected by unknown inputs, such as disturbances, faults or unmodeled dynamics. In this paper, we consider the problem of simultaneously estimating the state and unknown inputs in TS systems. The inputs considered in this paper are 1) polynomials in time (such as a bias in the model or an unknown ramp input acting on the model) and 2) unmodeled dynamics. The proposed observer is designed based on the known part of the fuzzy model. Conditions on the asymptotic convergence of the observer are presented and the design guarantees an ultimate bound on the error signal. The results are illustrated on a simulation example.

Key words: TS fuzzy systems, fuzzy observers, Lyapunov stability, adaptive observers, unknown input observers.

${ }^{*}$ Corresponding author. Tel.:+31 (0)15 27 88573, Fax:+31 (0)15 2786679

Email addresses: z.lendek@tudelft.nl (Zs. Lendek), jimmy.lauber@univ-valenciennes.fr (J. Lauber), thierry.guerra@univ-valenciennes.fr (T. M. Guerra), r.babuska@tudelft.nl (R. Babuška), b.deschutter@tudelft.nl (B. De Schutter)
\end{abstract}




\section{Introduction}

Many problems in decision making, control, and monitoring require the estimation of states and possibly uncertain parameters, based on a dynamic system model and a sequence of noisy measurements. For such a purpose, dynamic systems are often modeled in the state-space framework, using the state-transition model, which describes the evolution of states over time, and the sensor model, which relates the measurements to the states.

A generic method for the design of an observer valid for all types of nonlinear systems has not yet been developed. Moreover, a large class of nonlinear systems can be well approximated by TS fuzzy models [1], which in theory can approximate a general nonlinear system to an arbitrary degree of accuracy [2]. Stability conditions have been derived for TS fuzzy systems, most of them relying on the feasibility of an associated system of linear matrix inequalities (LMIs) [3-5]. A comprehensive survey on the analysis of fuzzy systems can be found in [6].

For a TS fuzzy model, well-established methods and algorithms can be used to design observers that estimate unmeasurable states. Several types of such observers have been developed for TS fuzzy systems, among which: fuzzy ThauLuenberger observers [3, 7], reduced-order observers [5, 8], and sliding-mode observers [9]. In general, the design methods for observers also lead to an LMI feasibility problem.

Adaptive observers are observers that simultaneously estimate the states and unknown parameters, by processing the measurements online. For SISO LTI systems, the adaptive observer design has been largely investigated (see [10] and the references therein).

A general tendency in case of nonlinear systems, both in adaptive controller and observer design [11-15] is to assume that the system is SISO and in observer canonical form. By using a quadratic Lyapunov function, ensuring strictly positive real conditions, the Kalman-Yakubovic-Popov lemma is applied and the adaptive laws are deduced from the Lyapunov synthesis. A shortcoming of these observers is that they do not incorporate prior information (such as an 
approximate model) and that they cannot be used when physical states have to be estimated, or when a model is not in a canonical form. Robust versions of these adaptive observers have also been derived for systems affected by a bounded disturbance, by adding a robustness term [11, 13, 15-17]. In many cases, when using both an observer and a controller, the robustness term is incorporated in the controller instead, to deal with the approximation error and disturbances [12, 14, 18], [19], amended in [20]. Results for MIMO systems include high gain observers [21], special observer canonical forms of the system [22, 23], linearly parameterized neural networks [24, 25] and observers based on a known linear part of the model [26]. A linear observer applied for a nonlinear system in general can be used only in a small neighborhood of the linearization point. Moreover, such an observer can only deal with constant or slowly varying inputs [27].

In this paper, we consider polynomial unknown inputs (such as a bias in the model or an unknown ramp input acting on the model) and uncertainty in the model dynamics. Model-based observer design in the presence of bounded unknown inputs has been investigated recently [28-32]. However, these approaches estimate only the states, and attenuate the effect of the disturbances, but do not estimate the unknown inputs. Our objective is to estimate both the state and the unknown input from the available input and output information. The design of observers to estimate unknown inputs is an important problem, since in many cases not all the inputs are known [27, 31, 32], but is needed for proper planning and control. For instance, in machine tool and manipulator applications, the cutting force exerted by the tool or the exerting force/torque of the robot is of interest, but it is very difficult or expensive to measure [26, 33]. Load estimation in e.g., electricity distribution networks [34], or wind turbines [35] is necessary for proper planning and operation. In biomechanics, the myoskeletal system can be regarded as a dynamic system, where segment positions and trajectories are the system outputs and joint torques are the non-measurable inputs [36]. In traffic control, time-varying parameters have to be tracked, which can be regarded as unknown inputs [37]. In chaotic systems, for chaos synchronization 
and secure communication, one has to estimate not only the state, but also the input signal[38]. The class of adaptive observers has received considerable interest in fault detection and identification, where the unknown inputs represent the effect of actuator failures or plant components and its presence has to be detected as soon as possible. However, many of these methods only detect the fault or attenuate its effect on the states [39], and rarely reconstruct it [40].

We develop a method to design observers for TS fuzzy systems with polynomial unknown inputs or unmodeled dynamics. The idea behind this type of design is that, in practice, a TS representation of a nonlinear system may be obtained by identifying LTI models in different operation points. However, such models are often inaccurate. By estimating the unknown inputs or unmodeled dynamics, the accuracy of the identified models can be improved. The observers are designed based on the already identified model, such that, together with the appropriate update law estimating the unknown inputs, they ensure the convergence of the estimation error.

The structure of the paper is as follows. Section 2 reviews stability conditions for TS fuzzy systems and observers. Section 3 presents the model and the observer considered. The proposed design for observers in the case of polynomial unknown inputs is described in Section 4. Section 5 presents the observer for estimating unmodeled dynamics. Related work is presented in Section 6. An example is given in Section 7. Finally, Section 8 concludes the paper.

\section{Stability of Fuzzy Systems and Observers}

Though the consequents of dynamic TS fuzzy systems are usually chosen linear or affine, it is well-known that the stability of these local models does not imply the stability of the overall fuzzy system. In the full fuzzy model, the local models are blended, and the particular blending of several local models may be strongly nonlinear, which influences the stability. Several stability conditions have been derived to ensure the stability of the system for any normalized membership function, independent of the operators used in the antecedent rules [3]. 
Most of these conditions depend on the feasibility of an associated LMI problem. In this section, we introduce the notations used throughout the paper and review some conditions for the stability of fuzzy systems and observer design. Throughout the paper it is assumed that the membership functions of the TS system are normalized.

\subsection{Preliminaries}

Throughout the paper $I$ denotes the identity matrix of the appropriate dimension, $\mathcal{H}(A)$ denotes the Hermitian of the matrix $A$, i.e., $\mathcal{H}(A)=A+A^{T}$.

Definition 1. Uniform ultimate boundedness [41] The solutions of $\dot{\boldsymbol{x}}=\boldsymbol{f}(t, \boldsymbol{x})$ are uniformly ultimately bounded with ultimate bound $b$ if there exist $b, c>0$ and for every $0<a<c$, there exists $T=T(a, b) \geq 0$ such that

$$
\left\|\boldsymbol{x}\left(t_{0}\right)\right\| \leq a \Longrightarrow\|\boldsymbol{x}(t)\| \leq b \forall t \geq t_{0}+T
$$

\subsection{Autonomous Fuzzy Systems}

Consider the autonomous fuzzy system:

$$
\dot{\boldsymbol{x}}=\sum_{i=1}^{m} w_{i}(\boldsymbol{z}) A_{i} \boldsymbol{x}
$$

where $A_{i}, i=1,2, \ldots, m$ represents the $i$ th local linear model, $w_{i}$ is the corresponding normalized membership function, and $z$ the vector of the scheduling variables.

For system (1), several stability conditions have been derived. Among them, a well-known and frequently used condition is formulated below [3]

Theorem 1. System (1) is exponentially stable if there exists $P=P^{T}>0$ so that $\mathcal{H}\left(P A_{i}\right)<0$, for $i=1,2, \ldots, m$. 
Remark: Less conservative, but more complex conditions can be obtained by using relaxations for LMIs $[3,6,42,43]$.

A condition on the convergence rate of system (1) was also derived from Theorem 1 [3].

Theorem 2. The decay rate of system (1) is at least $\alpha$, if there exists $P=$ $P^{T}>0$, so that

$$
\mathcal{H}\left(P A_{i}\right)+2 \alpha P<0 \quad i=1,2, \ldots, m
$$

Stability conditions similar to those of Theorem 1 can be used if the system considered is subjected to disturbances. Consider the following perturbed fuzzy system:

$$
\dot{\boldsymbol{x}}=\sum_{i=1}^{m} w_{i}(\boldsymbol{z}) A_{i} \boldsymbol{x}+D \boldsymbol{f}(t, \boldsymbol{x})
$$

where $D$ is a perturbation distribution matrix and $\boldsymbol{f}$ is Lipschitz in $\boldsymbol{x}$, i.e., there exists $\mu>0$ so that $\|\boldsymbol{f}(t, \boldsymbol{x})\| \leq \mu\|\boldsymbol{x}\|$, for all $t$ and $\boldsymbol{x}$. With these assumptions, a sufficient stability condition can be formalized by the following theorem [42].

Theorem 3. System (2) is exponentially stable if there exist matrices $P=P^{T}$, $Q=Q^{T}$, so that

$$
\begin{aligned}
& P>0 \quad Q>0 \\
& \mu \leq \frac{\lambda_{\min }(Q)}{\|P D\|} \\
& \mathcal{H}\left(P A_{i}\right)<-2 Q \quad i=1,2, \ldots, m
\end{aligned}
$$

where $\lambda_{\min }$ is the smallest eigenvalue.

Several variants of the above theorem exist, together with algorithms to compute robustness measures [42].

Other approaches, based on partitioning the state-space into regions determined by simultaneously active membership functions can be found in $[4,44,45]$. Similar conditions for the discrete-time case are described in [46]. 


\subsection{Fuzzy Observers}

Consider now the affine fuzzy system

$$
\begin{aligned}
\dot{\boldsymbol{x}} & =\sum_{i=1}^{m} w_{i}(\boldsymbol{z})\left(A_{i} \boldsymbol{x}+B_{i} \boldsymbol{u}+a_{i}\right) \\
\boldsymbol{y} & =\sum_{i=1}^{m} w_{i}(\boldsymbol{z}) C_{i} \boldsymbol{x}
\end{aligned}
$$

and an observer of the form

$$
\begin{aligned}
& \dot{\hat{\boldsymbol{x}}}=\sum_{i=1}^{m} w_{i}(\widehat{\boldsymbol{z}})\left(A_{i} \widehat{\boldsymbol{x}}+B_{i} \boldsymbol{u}+a_{i}+L_{i}(\boldsymbol{y}-\widehat{\boldsymbol{y}})\right) \\
& \widehat{\boldsymbol{y}}=\sum_{i=1}^{m} w_{i}(\widehat{\boldsymbol{z}}) C_{i} \widehat{\boldsymbol{x}} .
\end{aligned}
$$

As before, it is assumed that the membership functions are normalized. Depending on the explicit form of the error dynamics given by $\dot{\boldsymbol{e}}=\dot{\boldsymbol{x}}-\dot{\widehat{\boldsymbol{x}}}$, the theorems presented in Section 2.2 can be directly applied, or similar conditions may be derived to ensure the convergence to zero of the estimation error. For the design, two cases are distinguished: 1) the scheduling vector $\boldsymbol{z}$ does not depend on the estimated states, i.e., $\widehat{\boldsymbol{z}}=\boldsymbol{z}$ and 2) $\widehat{\boldsymbol{z}}$ depends on (some of) the states to be estimated.

\subsubsection{The scheduling vector does not depend on the estimated states}

In this case, the error system can be written as:

$$
\begin{aligned}
\dot{\boldsymbol{e}} & =\sum_{i=1}^{m} \sum_{j=1}^{m} w_{i}(\boldsymbol{z}) w_{j}(\boldsymbol{z})\left(A_{i}-L_{i} C_{j}\right) \boldsymbol{e} \\
& =\sum_{i=1}^{m} w_{i}(\boldsymbol{z}) w_{i}(\boldsymbol{z})\left(A_{i}-L_{i} C_{i}\right) \boldsymbol{e}+\sum_{i=1}^{m} \sum_{\substack{j=1 \\
j>i}}^{m} w_{i}(\boldsymbol{z}) w_{j}(\boldsymbol{z})\left(A_{i}-L_{i} C_{j}+A_{j}-L_{j} C_{i}\right) \boldsymbol{e} .
\end{aligned}
$$

Using a Lyapunov function of the form $V(t)=\boldsymbol{e}^{T} P \boldsymbol{e}$, with $P=P^{T}>0$, basic sufficient stability conditions for this system were derived in [3]:

Theorem 4. The system (6) is GAS, if there exists $P=P^{T}>0$ so that for $i=1,2, \ldots, m$ and $j=1,2, \ldots, m$ :

$$
\mathcal{H}\left(P\left(A_{i}-L_{i} C_{j}+A_{j}-L_{j} C_{i}\right)\right)<0 \quad \forall j \geq i: \quad \exists \boldsymbol{z}: \quad w_{i}(\boldsymbol{z}) w_{j}(\boldsymbol{z}) \neq 0
$$


A well-known condition on the design of the observer for the system (1), so that a desired convergence rate $\alpha$ is guaranteed, has also been obtained in [3] by appropriately modifying Theorem 2 .

\subsubsection{The scheduling vector depends on the estimated states}

The second case is when the scheduling vector depends on the states to be estimated. For the simplicity of the notation, only the case with common measurement matrices $\left(C_{i}=C, i=1,2, \ldots, m\right)$ is presented. Then, the observer (5) becomes:

$$
\begin{aligned}
\dot{\widehat{\boldsymbol{x}}} & =\sum_{i=1}^{m} w_{i}(\widehat{\boldsymbol{z}})\left(A_{i} \widehat{\boldsymbol{x}}+B_{i} \boldsymbol{u}+a_{i}+L_{i}(\boldsymbol{y}-\widehat{\boldsymbol{y}})\right) \\
\widehat{\boldsymbol{y}} & =C \widehat{\boldsymbol{x}}
\end{aligned}
$$

and the error dynamics can be expressed as:

$$
\dot{\boldsymbol{e}}=\sum_{i=1}^{m} w_{i}(\widehat{\boldsymbol{z}})\left(A_{i}-L_{i} C\right) \boldsymbol{e}+\sum_{i=1}^{m}\left(w_{i}(\boldsymbol{z})-w_{i}(\widehat{\boldsymbol{z}})\right)\left(A_{i} \boldsymbol{x}+B_{i} \boldsymbol{u}+a_{i}\right)
$$

For such a system, sufficient stability conditions are given by the following theorem [42].

Theorem 5. The error dynamics (9) is exponentially stable, if there exist $\mu>$ $0, P=P^{T}>0, Q=Q^{T}>0$ so that for all $i=1, \ldots, m$

$$
\begin{aligned}
\mathcal{H}\left(P\left(A_{i}-L_{i} C\right)\right) & \leq-Q \\
\left(\begin{array}{cc}
Q-\mu^{2} I & P \\
P & I
\end{array}\right) & >0 \\
\left\|\sum_{i=1}^{m}\left(w_{i}(\boldsymbol{z})-w_{i}(\widehat{\boldsymbol{z}})\right)\left(A_{i} \boldsymbol{x}+B_{i} \boldsymbol{u}+a_{i}\right)\right\| & \leq \mu\|e\|
\end{aligned}
$$

i.e., $\sum_{i=1}^{m}\left(w_{i}(\boldsymbol{z})-w_{i}(\widehat{\boldsymbol{z}})\right)\left(A_{i} \boldsymbol{x}+B_{i} \boldsymbol{u}+a_{i}\right)$ is Lipschitz in $\boldsymbol{e}$.

Note that since fuzzy systems are generally defined on a compact set, the condition that "there exists $\mu>0$ so that $\left\|\sum_{i=1}^{m}\left(w_{i}(\boldsymbol{z})-w_{i}(\widehat{\boldsymbol{z}})\right)\left(A_{i} \boldsymbol{x}+B_{i} \boldsymbol{u}+a_{i}\right)\right\| \leq$ 
$\mu\|e\| "$ is satisfied if $w_{i}(\boldsymbol{z}), i=1,2, \ldots, m$ are differentiable w.r.t. $\boldsymbol{x}$ almost everywhere and have a bounded first derivative for almost all $\boldsymbol{x}$. In practice, most membership functions used satisfy this condition, which is therefore not restrictive.

\section{Problem statement}

Consider the following fuzzy system:

$$
\begin{aligned}
& \dot{\boldsymbol{x}}=\sum_{i=1}^{m} w_{i}(\boldsymbol{z})\left(A_{i} \boldsymbol{x}+B_{i} \boldsymbol{u}+a_{i}+M_{i} \boldsymbol{d}\right) \\
& \boldsymbol{y}=\sum_{i=1}^{m} w_{i}(\boldsymbol{z}) C_{i} \boldsymbol{x}
\end{aligned}
$$

where $A_{i}, B_{i}, M_{i}, a_{i}, C_{i}, i=1,2, \ldots, m$ are known and the vector $\boldsymbol{d}$ is an unknown input. Design of unknown input observers is very important in practice, since, in many cases, not all the inputs are known. Moreover, these inputs can represent disturbances acting on the process, effects of uncertain dynamics or actuator failures. In this paper we consider two types of unknown inputs: 1) polynomials in time and 2) unmodelled dynamics of some of the states. Our goal is to design a stable observer to estimate simultaneously both the state vector $\boldsymbol{x}$ and the unknown inputs $\boldsymbol{d}$.

The observer considered is of the form:

$$
\begin{aligned}
\dot{\hat{\boldsymbol{x}}} & =\sum_{i=1}^{m} w_{i}(\widehat{\boldsymbol{z}})\left(A_{i} \widehat{\boldsymbol{x}}+B_{i} \boldsymbol{u}+a_{i}+M_{i} \widehat{\boldsymbol{d}}+L_{i}(\boldsymbol{y}-\widehat{\boldsymbol{y}})\right) \\
\widehat{\boldsymbol{y}} & =\sum_{i=1}^{m} w_{i}(\widehat{\boldsymbol{z}}) C_{i} \widehat{\boldsymbol{x}} \\
\dot{\hat{\boldsymbol{d}}} & =f(\widehat{\boldsymbol{d}}, \boldsymbol{w}(\widehat{\boldsymbol{z}}), \widehat{\boldsymbol{x}}, \boldsymbol{y})
\end{aligned}
$$

where $L_{i}, i=1,2, \ldots, m$, are the gain matrices to be designed for each rule, and $f$, the update law for $\boldsymbol{d}$, should be determined so that the estimation errors $\boldsymbol{x}-\widehat{\boldsymbol{x}}$ and $\boldsymbol{d}-\widehat{\boldsymbol{d}}$ converge asymptotically to zero.

Two main cases can be distinguished, depending on whether or not the scheduling vector depends on the states to be estimated. The observer design is considered in both cases. 


\section{Polynomial unknown inputs}

In this section, we consider the case when the unknown input is or can be approximated by a polynomial function in time. Such inputs may represent biases in the model, time-varying disturbances acting on the process, or the degradation in time or even failure of actuators. We give conditions to design a fuzzy observer and we also give a bound on the estimation error.

\subsection{Observer Design}

Consider the TS fuzzy system of the form

$$
\begin{aligned}
& \dot{\boldsymbol{x}}=\sum_{i=1}^{m} w_{i}(\boldsymbol{z})\left(A_{i} \boldsymbol{x}+B_{i} \boldsymbol{u}+M_{i} \boldsymbol{d}+a_{i}\right) \\
& \boldsymbol{y}=\sum_{i=1}^{m} w_{i}(\boldsymbol{z}) C_{i} \boldsymbol{x}
\end{aligned}
$$

where there exists $p \in \mathbb{N}$ so that $\boldsymbol{d}^{(p)}=0$, i.e., the unknown input is a $p-1$-th order polynomial in time. It is assumed that the states, the unknown input $\boldsymbol{d}$, and the derivatives of $\boldsymbol{d}$ are observable from $\boldsymbol{y}$.

\subsubsection{Case 1}

If $\boldsymbol{z}$ does not depend on $\widehat{\boldsymbol{x}}$, then

Theorem 6. The estimation error using the observer

$$
\begin{aligned}
\dot{\hat{\boldsymbol{x}}} & =\sum_{i=1}^{m} w_{i}(\boldsymbol{z})\left[A_{i} \widehat{\boldsymbol{x}}+B_{i} \boldsymbol{u}+L_{i}(\boldsymbol{y}-\widehat{\boldsymbol{y}})+M_{i} \widehat{\boldsymbol{d}}+a_{i}\right] \\
\widehat{\boldsymbol{y}} & =\sum_{i=1}^{m} w_{i}(\boldsymbol{z}) C_{i} \widehat{\boldsymbol{x}} \\
\widehat{\boldsymbol{d}}^{(p)} & =\sum_{i=1}^{m} w_{i}(\boldsymbol{z}) \Lambda_{i}^{p}(\boldsymbol{y}-\widehat{\boldsymbol{y}}) \\
\widehat{\boldsymbol{d}}^{(k)} & =\sum_{i=1}^{m} w_{i}(\boldsymbol{z})\left(\Lambda_{i}^{k}(\boldsymbol{y}-\widehat{\boldsymbol{y}})+\widehat{\boldsymbol{d}}^{(k+1)}\right) \\
& \text { for } k=1, \ldots, p-1
\end{aligned}
$$


is exponentially stable if there exist $P=P^{T}>0, L_{i}, \Lambda_{i}^{k}, i=1,2, \ldots, m$, $j=1,2, \ldots, m, k=1,2, \ldots, p$ so that

$$
\mathcal{H}\left(P\left(\begin{array}{ccccc}
A_{i}-L_{i} C_{j}+A_{j}-L_{j} C_{i} & M_{i}+M_{j} & 0 & \cdots & 0 \\
-\Lambda_{i}^{1} C_{j}-\Lambda_{j}^{1} C_{i} & 0 & 2 I & \cdots & 0 \\
\vdots & \vdots & \vdots & \ddots & \vdots \\
-\Lambda_{i}^{p-1} C_{j}-\Lambda_{j}^{p-1} C_{i} & 0 & 0 & \cdots & 2 I \\
-\Lambda_{i}^{p} C_{j}-\Lambda_{j}^{p} C_{i} & 0 & 0 & \cdots & 0
\end{array}\right)\right)<0
$$

$\forall j \geq i: \quad \exists \boldsymbol{z}: \quad w_{i}(\boldsymbol{z}) w_{j}(\boldsymbol{z}) \neq 0$

Proof: An extended error system, containing both the state error and the derivatives of the input error $\overline{\boldsymbol{d}}=\boldsymbol{d}-\widehat{\boldsymbol{d}}$, can be expressed as:

$$
\begin{aligned}
& \dot{\boldsymbol{e}}_{a}=\left(\begin{array}{c}
\dot{\boldsymbol{e}} \\
\dot{\overline{\boldsymbol{d}}} \\
\ddot{\overline{\boldsymbol{d}}} \\
\vdots \\
\overline{\boldsymbol{d}}^{(p)}
\end{array}\right)=\sum_{i=1}^{m} w_{i}(\boldsymbol{z}) w_{i}(\boldsymbol{z})\left(\begin{array}{ccccc}
A_{i}-L_{i} C_{i} & M_{i} & 0 & \cdots & 0 \\
-\Lambda_{i}^{1} C_{i} & 0 & I & \cdots & 0 \\
\vdots & \vdots & \vdots & \ddots & \vdots \\
-\Lambda_{i}^{p-1} C_{i} & 0 & 0 & \cdots & I \\
-\Lambda_{i}^{p} C_{i} & 0 & 0 & \cdots & 0
\end{array}\right)\left(\begin{array}{c}
\boldsymbol{e} \\
\overline{\boldsymbol{d}} \\
\vdots \\
\overline{\boldsymbol{d}}^{(p-1)}
\end{array}\right) \\
& +\sum_{i=1}^{m} w_{i}(\boldsymbol{z}) \sum_{\substack{j=1 \\
j>i}}^{m} w_{j}(\boldsymbol{z})\left(\begin{array}{ccccc}
A_{i}-L_{i} C_{j}+A_{j}-L_{j} C_{i} & M_{i}+M_{j} & 0 & \cdots & 0 \\
-\Lambda_{i}^{1} C_{j}-\Lambda_{j}^{1} C_{i} & 0 & 2 I & \cdots & 0 \\
\vdots & \vdots & \vdots & \ddots & \vdots \\
-\Lambda_{i}^{p-1} C_{j}-\Lambda_{j}^{p-1} C_{i} & 0 & 0 & \cdots & 2 I \\
-\Lambda_{i}^{p} C_{j}-\Lambda_{j}^{p} C_{i} & 0 & 0 & \cdots & 0
\end{array}\right)\left(\begin{array}{c}
\boldsymbol{e} \\
\overline{\boldsymbol{d}} \\
\vdots \\
\overline{\boldsymbol{d}}^{(p-1)}
\end{array}\right)
\end{aligned}
$$


Using a quadratic Lyapunov function for the extended error vector $V=$ $\boldsymbol{e}_{a}^{T} P \boldsymbol{e}_{a}$, the derivative is expressed as:

$$
\begin{aligned}
& \dot{V}=\sum_{i=1}^{m} w_{i}(\boldsymbol{z}) w_{i}(\boldsymbol{z}) \boldsymbol{e}_{a}^{T} \mathcal{H}\left(P\left(\begin{array}{ccccc}
A_{i}-L_{i} C_{i} & M_{i} & 0 & \cdots & 0 \\
-\Lambda_{i}^{1} C_{i} & 0 & I & \cdots & 0 \\
\vdots & \vdots & \vdots & \ddots & \vdots \\
-\Lambda_{i}^{p-1} C_{i} & 0 & 0 & \cdots & I \\
-\Lambda_{i}^{p} C_{i} & 0 & 0 & \cdots & 0
\end{array}\right)\right) \boldsymbol{e}_{a} \\
& +\sum_{i=1}^{m} w_{i}(\boldsymbol{z}) \sum_{\substack{j=1 \\
j>i}}^{m} w_{j}(\boldsymbol{z}) \boldsymbol{e}_{a}^{T} \mathcal{H}\left(P\left(\begin{array}{ccccc}
A_{i}-L_{i} C_{j}+A_{j}-L_{j} C_{i} & M_{i}+M_{j} & 0 & \ldots & 0 \\
-\Lambda_{i}^{1} C_{j}-\Lambda_{j}^{1} C_{i} & 0 & 2 I & \ldots & 0 \\
\vdots & \vdots & \vdots & \ddots & \vdots \\
-\Lambda_{i}^{p-1} C_{j}-\Lambda_{j}^{p-1} C_{i} & 0 & 0 & \ldots & 2 I \\
-\Lambda_{i}^{p} C_{j}-\Lambda_{j}^{p} C_{i} & 0 & 0 & \ldots & 0
\end{array}\right)\right) \boldsymbol{e}_{a}
\end{aligned}
$$

which is negative definite if the condition (15) is satisfied.

Remark: In order to design observers with a desired convergence rate $\alpha$, Theorem 2 can be applied. The estimation error of the observer (14) converges with a rate at least $\alpha$ if there exists $P=P^{T}>0, L_{i}, \Lambda_{i}^{k}, i=1,2, \ldots, m$, $j=1,2, \ldots, m, k=1,2, \ldots, p$ so that

$$
\mathcal{H}\left(P\left(\begin{array}{ccccc}
A_{i}-L_{i} C_{j}+A_{j}-L_{j} C_{i} & M_{i}+M_{j} & 0 & \cdots & 0 \\
-\Lambda_{i}^{1} C_{j}-\Lambda_{j}^{1} C_{i} & 0 & 2 I & \cdots & 0 \\
\vdots & \vdots & \vdots & \ddots & \vdots \\
-\Lambda_{i}^{p-1} C_{j}-\Lambda_{j}^{p-1} C_{i} & 0 & 0 & \cdots & 2 I \\
-\Lambda_{i}^{p} C_{j}-\Lambda_{j}^{p} C_{i} & 0 & 0 & \cdots & 0
\end{array}\right)\right)+4 \alpha P<0
$$

$\forall j \geq i: \quad \exists \boldsymbol{z}: \quad w_{i}(\boldsymbol{z}) w_{j}(\boldsymbol{z}) \neq 0$

The proof follows directly.

\subsubsection{Case 2}

A similar observer can also be designed if $\boldsymbol{z}$ depends on $\widehat{\boldsymbol{x}}$. For the simplicity of the notation, only the case when the measurement matrix is common for all 
rules is presented. Note, however, that if the measurement matrices are different for the rules, the observer can be designed analogously.

The observer considered is of the form

$$
\begin{aligned}
\dot{\widehat{\boldsymbol{x}}} & =\sum_{i=1}^{m} w_{i}(\widehat{\boldsymbol{z}})\left[A_{i} \widehat{\boldsymbol{x}}+B_{i} \boldsymbol{u}+L_{i}(\boldsymbol{y}-\widehat{\boldsymbol{y}})+M_{i} \widehat{\boldsymbol{d}}+a_{i}\right] \\
\widehat{\boldsymbol{y}} & =C \widehat{\boldsymbol{x}} \\
\widehat{\boldsymbol{d}}^{(p)} & =\sum_{i=1}^{m} w_{i}(\widehat{\boldsymbol{z}}) \Lambda_{i}^{p}(\boldsymbol{y}-\widehat{\boldsymbol{y}}) \\
\widehat{\boldsymbol{d}}^{(j)} & =\sum_{i=1}^{m} w_{i}(\widehat{\boldsymbol{z}})\left(\Lambda_{i}^{j}(\boldsymbol{y}-\widehat{\boldsymbol{y}})+\widehat{\boldsymbol{d}}^{(j+1)}\right) \\
& \text { for } j=1, \ldots, p-1
\end{aligned}
$$

Then the extended error system becomes:

$$
\begin{aligned}
& \dot{\boldsymbol{e}}_{a}=\sum_{i=1}^{m} w_{i}(\widehat{\boldsymbol{z}})\left(\begin{array}{ccccc}
A_{i}-L_{i} C & M_{i} & 0 & \cdots & 0 \\
-\Lambda_{i}^{1} C & 0 & I & \cdots & 0 \\
\vdots & \vdots & \vdots & \ddots & \vdots \\
-\Lambda_{i}^{p-1} C & 0 & 0 & \cdots & I \\
-\Lambda_{i}^{p} C & 0 & 0 & \cdots & 0
\end{array}\right)\left(\begin{array}{c}
\boldsymbol{e} \\
\overline{\boldsymbol{d}} \\
\vdots \\
\overline{\boldsymbol{d}}^{(p-1)}
\end{array}\right) \\
& +\sum_{i=1}^{m}\left(w_{i}(\boldsymbol{z})-w_{i}(\widehat{\boldsymbol{z}})\right)\left(\begin{array}{llll}
I & 0 & \ldots & 0
\end{array}\right)^{T} \cdot\left(A_{i} \boldsymbol{x}+B_{i} \boldsymbol{u}+M_{i} \boldsymbol{d}+a_{i}\right)
\end{aligned}
$$

Assuming that $\left\|\sum_{i=1}^{m}\left(w_{i}(\boldsymbol{z})-w_{i}(\widehat{\boldsymbol{z}})\right)\left(A_{i} \boldsymbol{x}+B_{i} \boldsymbol{u}+M_{i} \boldsymbol{d}+a_{i}\right)\right\| \leq \mu\|\boldsymbol{e}\|$, and therefore it is also Lipschitz in $\boldsymbol{e}_{a}$, with the same Lipschitz constant, the stability conditions become:

Corollary 1. The error system (19) is asymptotically stable, if there exist $P=$ 
$P^{T}>0, Q=Q^{T}>0, L_{i}, \Lambda_{i}^{j}, i=1,2, \ldots, m, j=1,2, \ldots, p$ so that

$$
\begin{aligned}
& \mathcal{H}\left(P\left(\begin{array}{ccccc}
A_{i}-L_{i} C & M_{i} & 0 & \cdots & 0 \\
-\Lambda_{i}^{1} C & 0 & I & \cdots & 0 \\
\vdots & \vdots & \vdots & \ddots & \vdots \\
-\Lambda_{i}^{p-1} C & 0 & 0 & \cdots & I \\
-\Lambda_{i}^{p} C & 0 & 0 & \cdots & 0
\end{array}\right)\right)<-Q \\
& \left\|\sum_{i=1}^{m}\left(w_{i}(\boldsymbol{z})-w_{i}(\widehat{\boldsymbol{z}})\right)\left(A_{i} \boldsymbol{x}+B_{i} \boldsymbol{u}+M_{i} \boldsymbol{d}+a_{i}\right)\right\| \leq \mu\|e\| \\
& \left(\begin{array}{cc}
Q-\mu^{2} I & P \\
P & I
\end{array}\right)>0
\end{aligned}
$$

The proof follows directly.

\subsection{Bound on Errors}

In most cases, the unknown input is not polynomial, but it is possible to determine a bound on some derivative of it. Therefore, consider the case when there exists $p \in \mathbb{N}$ so that $\boldsymbol{d}^{(p)}$ is bounded by a known constant, i.e., $\left\|\boldsymbol{d}^{(p)}\right\|<\mu_{\boldsymbol{d}}$, and $\boldsymbol{d}^{(j)}, j=1,2, \ldots, p$ are observable from $\boldsymbol{y}$. In this case, although the estimation error does not converge to zero, it is bounded, and an upper bound on it can be computed as follows. 


\subsubsection{Case 1}

If the scheduling vector does not depend on $\widehat{\boldsymbol{x}}$, the error system can be written as:

$$
\begin{aligned}
\dot{\boldsymbol{e}}_{a}=\sum_{i=1}^{m} w_{i}(\boldsymbol{z}) w_{i}(\boldsymbol{z})\left(\begin{array}{ccccc}
A_{i}-L_{i} C_{i} & M_{i} & 0 & \cdots & 0 \\
-\Lambda_{i}^{1} C_{i} & 0 & I & \cdots & 0 \\
\vdots & \vdots & \vdots & \ddots & \vdots \\
-\Lambda_{i}^{p-1} C_{i} & 0 & 0 & \cdots & I \\
-\Lambda_{i}^{p} C_{i} & 0 & 0 & \cdots & 0
\end{array}\right) \boldsymbol{e}_{a}+\left(\begin{array}{c}
0 \\
0 \\
0 \\
\vdots \\
\boldsymbol{d}^{(p)}
\end{array}\right) \\
+\sum_{i=1}^{m} w_{i}(\boldsymbol{z}) \sum_{j=1}^{m} w_{j}(\boldsymbol{z})\left(\begin{array}{ccccc}
A_{i}-L_{i} C_{j}+A_{j}-L_{j} C_{i} & M_{i}+M_{j} & 0 & \cdots & 0 \\
-\Lambda_{i}^{1} C_{j}-\Lambda_{j}^{1} C_{i} & 0 & 2 I & \cdots & 0 \\
\vdots & \vdots & \vdots & \ddots & \vdots \\
-\Lambda_{i}^{p-1} C_{j}-\Lambda_{j}^{p-1} C_{i} & 0 & 0 & \cdots & 2 I \\
-\Lambda_{i}^{p} C_{j}-\Lambda_{j}^{p} C_{i} & 0 & 0 & \cdots & 0
\end{array}\right) \boldsymbol{e}_{a}
\end{aligned}
$$

Theorem 7. The error described by (21) is ultimately bounded by a ball with radius

$$
\gamma=\sqrt{\frac{\lambda_{\max }(P)}{\lambda_{\min }(P)}} \frac{\lambda_{\max }(P) \mu_{d}}{\sigma \lambda_{\min }(Q)}
$$

if there exist $P=P^{T}>0, Q=Q^{T}>0, L_{i}, \Lambda_{i}^{k}, i=1,2, \ldots, m, k=$ $1,2, \ldots, p, j=1,2, \ldots, m$ so that

$$
\begin{aligned}
& \left\|\boldsymbol{d}^{(p)}\right\|<\mu_{\boldsymbol{d}} \\
& \mathcal{H}\left(P\left(\begin{array}{ccccc}
A_{i}-L_{i} C_{j}+A_{j}-L_{j} C_{i} & M_{i}+M_{j} & 0 & \cdots & 0 \\
-\Lambda_{i}^{1} C_{j}-\Lambda_{j}^{1} C_{i} & 0 & 2 I & \cdots & 0 \\
\vdots & \vdots & \vdots & \ddots & \vdots \\
-\Lambda_{i}^{p-1} C_{j}-\Lambda_{j}^{p-1} C_{i} & 0 & 0 & \cdots & 2 I \\
-\Lambda_{i}^{p} C_{j}-\Lambda_{j}^{p} C_{i} & 0 & 0 & \cdots & 0
\end{array}\right)\right)<-4 Q
\end{aligned}
$$

$$
\forall j \geq i: \quad \exists \boldsymbol{z}: \quad w_{i}(\boldsymbol{z}) w_{j}(\boldsymbol{z}) \neq 0
$$

for $i=1,2, \ldots, m$, where $\sigma \in(0,1)$ and $\lambda_{\min }$ and $\lambda_{\max }$ denote the eigenvalues with the smallest and largest absolute magnitude, respectively. 
Proof: Consider a quadratic Lyapunov function for the extended error vector, $V=\boldsymbol{e}_{a}^{T} P \boldsymbol{e}_{a}$. Then,

$$
\begin{aligned}
& \dot{V}=\sum_{i=1}^{m} w_{i}(\boldsymbol{z}) w_{i}(\boldsymbol{z}) \boldsymbol{e}_{a}^{T} \mathcal{H}\left(P\left(\begin{array}{ccccc}
A_{i}-L_{i} C_{i} & M_{i} & 0 & \cdots & 0 \\
-\Lambda_{i}^{1} C_{i} & 0 & I & \cdots & 0 \\
\vdots & \vdots & \vdots & \ddots & \vdots \\
-\Lambda_{i}^{p-1} C_{i} & 0 & 0 & \cdots & I \\
-\Lambda_{i}^{p} C_{i} & 0 & 0 & \cdots & 0
\end{array}\right)\right) \boldsymbol{e}_{a} \\
& +\sum_{i=1}^{m} w_{i}(\boldsymbol{z}) \sum_{\substack{j=1 \\
j>i}}^{m} w_{j}(\boldsymbol{z}) \boldsymbol{e}_{a}^{T} \mathcal{H}\left(P\left(\begin{array}{ccccc}
A_{i}-L_{i} C_{j}+A_{j}-L_{j} C_{i} & M_{i}+M_{j} & 0 & \ldots & 0 \\
-\Lambda_{i}^{1} C_{j}-\Lambda_{j}^{1} C_{i} & 0 & 2 I & \ldots & 0 \\
\vdots & \vdots & \vdots & \ddots & \vdots \\
-\Lambda_{i}^{p-1} C_{j}-\Lambda_{j}^{p-1} C_{i} & 0 & 0 & \ldots & 2 I \\
-\Lambda_{i}^{p} C_{j}-\Lambda_{j}^{p} C_{i} & 0 & 0 & \ldots & 0
\end{array}\right)\right) \boldsymbol{e}_{a} \\
& +\sum_{i=1}^{m} w_{i}(\boldsymbol{z}) 2 \boldsymbol{e}_{a}^{T} P\left(\begin{array}{lllll}
0 & 0 & 0 & \cdots & \boldsymbol{d}^{(p)^{T}}
\end{array}\right)^{T} \\
& \leq-2 \lambda_{\min }(Q)\left\|\boldsymbol{e}_{a}\right\|^{2}+2 \lambda_{\max }(P)\left\|\boldsymbol{e}_{a}\right\| \mu_{\boldsymbol{d}} \\
& \leq-2(1-\sigma) \lambda_{\min }(Q)\left\|\boldsymbol{e}_{a}\right\|^{2}-2\left(\sigma \lambda_{\min }(Q)\left\|\boldsymbol{e}_{a}\right\|^{2}-\lambda_{\max }(P)\left\|\boldsymbol{e}_{a}\right\| \mu_{\boldsymbol{d}}\right)
\end{aligned}
$$

where $\sigma \in(0,1)$ is arbitrarily chosen and $Q=Q^{T}$ is a positive definite matrix such that (23) is satisfied. Then, $\dot{V}$ is negative definite if

$$
\begin{aligned}
& \sigma \lambda_{\min }(Q)\left\|\boldsymbol{e}_{a}\right\|^{2}-\lambda_{\max }(P)\left\|\boldsymbol{e}_{a}\right\| \mu_{\boldsymbol{d}}>0 \\
& \left\|\boldsymbol{e}_{a}\right\|>\frac{\lambda_{\max }(P) \mu_{\boldsymbol{d}}}{\sigma \lambda_{\min }(Q)}
\end{aligned}
$$

Since $\lambda_{\min }(P)\left\|\boldsymbol{e}_{a}\right\|^{2} \leq V \leq \lambda_{\max }(P)\left\|\boldsymbol{e}_{a}\right\|^{2}$, using Theorem 4.18 of [41] it can be concluded that $\left\|e_{a}\right\|$ converges exponentially to a ball with radius

$$
\gamma=\sqrt{\frac{\lambda_{\max }(P)}{\lambda_{\min }(P)}} \frac{\lambda_{\max }(P) \mu_{\boldsymbol{d}}}{\sigma \lambda_{\min }(Q)}
$$

which is a global uniform ultimate bound on the estimation error [41].

Remark: This bound can be minimized by using the relaxation in [43] and solving the following optimization problem: 
find $P, L_{i}, \Lambda_{i}^{k}, i=1,2, \ldots, m, j=1,2, \ldots, m, k=1,2, \ldots, p$ and maximize $\alpha_{1}, \alpha_{2}, \alpha_{3}$ subject to:

$$
\begin{aligned}
& \Gamma_{i j}=\mathcal{H}\left(P\left(\begin{array}{ccccc}
A_{i}-L_{i} C_{j} & M_{i} & 0 & \cdots & 0 \\
-\Lambda_{i}^{1} C_{j} & 0 & I & \cdots & 0 \\
\vdots & \vdots & \vdots & \ddots & \vdots \\
-\Lambda_{i}^{p-1} C_{j} & 0 & 0 & \cdots & I \\
-\Lambda_{i}^{p} C_{j} & 0 & 0 & \cdots & 0
\end{array}\right)\right) \\
& P=P^{T}>0 \\
& \Gamma_{i i}>0 \\
& \frac{2}{m-1} \Gamma_{i i}+\Gamma_{i j}+\Gamma_{j i}<-\alpha_{3} I \\
& \forall i, j: \quad \exists \boldsymbol{z}: w_{i}(\boldsymbol{z}) w_{j}(\boldsymbol{z}) \neq 0 \\
& -P>-\alpha_{2} I \\
& P>\alpha_{1} I
\end{aligned}
$$

for all $i=1,2, \ldots, m$.

\subsubsection{Case 2}

A similar, though notably more conservative bound can be found in the case when $\boldsymbol{z}$ is a function of $\widehat{\boldsymbol{x}}$. For the simplicity of the notation, the computation is presented for the case when the measurement matrices are common for all the 
rules. The error system is that in (19) and let the condition

$$
\begin{aligned}
& \mathcal{H}\left(P\left(\begin{array}{ccccc}
A_{i}-L_{i} C & M_{i} & 0 & \cdots & 0 \\
-\Lambda_{i}^{1} C & 0 & I & \cdots & 0 \\
\vdots & \vdots & \vdots & \ddots & \vdots \\
-\Lambda_{i}^{p-1} C & 0 & 0 & \cdots & I \\
-\Lambda_{i}^{p} C & 0 & 0 & \cdots & 0
\end{array}\right)\right)<-2 Q \\
& P=P^{T}>0 \\
& Q=Q^{T}>0 \\
& \left\|\sum_{i=1}^{m}\left(w_{i}(\boldsymbol{z})-w_{i}(\widehat{\boldsymbol{z}})\right)\left(A_{i} \boldsymbol{x}+B_{i} \boldsymbol{u}+M_{i} \boldsymbol{d}+a_{i}\right)\right\| \leq \mu\|e\| \\
& \left(\begin{array}{cc}
Q-\mu^{2} I & P \\
P & I
\end{array}\right)>0
\end{aligned}
$$

hold. Using a quadratic Lyapunov function for the extended error vector, $V=$ $\boldsymbol{e}_{a}^{T} P \boldsymbol{e}_{a}$, we obtain

$$
\begin{aligned}
& \dot{V}=\sum_{i=1}^{m} w_{i}(\boldsymbol{z}) \boldsymbol{e}_{a}^{T} \mathcal{H}\left(P\left(\begin{array}{ccccc}
A_{i}-L_{i} C & M_{i} & 0 & \cdots & 0 \\
-\Lambda_{i}^{1} C & 0 & I & \cdots & 0 \\
\vdots & \vdots & \vdots & \ddots & \vdots \\
-\Lambda_{i}^{p-1} C & 0 & 0 & \cdots & I \\
-\Lambda_{i}^{p} C & 0 & 0 & \cdots & 0
\end{array}\right)\right) \boldsymbol{e}_{a} \\
& +\sum_{i=1}^{m} w_{i}(\boldsymbol{z}) 2 \boldsymbol{e}_{a}^{T} P\left(\begin{array}{lllll}
0 & 0 & 0 & \cdots & \boldsymbol{d}^{(p)^{T}}
\end{array}\right)^{T} \\
& +2 \boldsymbol{e}_{a}^{T} P \sum_{i=1}^{m}\left(\left(w_{i}(\boldsymbol{z})-w_{i}(\widehat{\boldsymbol{z}})\right)\right)\left(\begin{array}{llll}
I & 0 & \cdots & 0
\end{array}\right)^{T}\left(A_{i} \boldsymbol{x}+B_{i} \boldsymbol{u}+M_{i} \boldsymbol{d}\right) \\
& \leq-2 \lambda_{\min }(Q)\left\|\boldsymbol{e}_{a}\right\|^{2}+2 \lambda_{\max }(P) \mu\left\|\boldsymbol{e}_{a}\right\|^{2}+2 \lambda_{\max }(P)\left\|\boldsymbol{e}_{a}\right\| \mu_{\boldsymbol{d}} \\
& \leq-2(1-\sigma)\left(\lambda_{\min }(Q)-\mu \lambda_{\max }(P)\right)\left\|\boldsymbol{e}_{a}\right\|^{2}-2\left(\sigma\left(\lambda_{\min }(Q)-\mu \lambda_{\max }(P)\right)\left\|\boldsymbol{e}_{a}\right\|^{2}-\lambda_{\max }(P)\left\|\boldsymbol{e}_{a}\right\| \mu_{\boldsymbol{d}}\right)
\end{aligned}
$$


where $\sigma \in(0,1)$ is arbitrarily chosen and $Q=Q^{T}$ is a positive definite matrix such that (26) is satisfied. Then, $\dot{V}$ is negative definite if

$$
\begin{aligned}
& \sigma\left(\lambda_{\min }(Q)-\mu \lambda_{\max }(P)\right)\left\|\boldsymbol{e}_{a}\right\|^{2}-\lambda_{\max }(P)\left\|\boldsymbol{e}_{a}\right\| \mu_{\boldsymbol{d}}>0 \\
& \left\|\boldsymbol{e}_{a}\right\|>\frac{\lambda_{\max }(P) \mu_{\boldsymbol{d}}}{\sigma\left(\lambda_{\min }(Q)-\mu \lambda_{\max }(P)\right)}
\end{aligned}
$$

Since $\lambda_{\min }(P)\left\|\boldsymbol{e}_{a}\right\|^{2} \leq V \leq \lambda_{\max }(P)\left\|\boldsymbol{e}_{a}\right\|^{2}$, using Theorem 4.18 of [41] it can be concluded that $\left\|\boldsymbol{e}_{a}\right\|$ converges exponentially to a ball with radius

$$
\gamma=\sqrt{\frac{\lambda_{\max }(P)}{\lambda_{\min }(P)}} \frac{\lambda_{\max }(P) \mu_{\boldsymbol{d}}}{\sigma\left(\lambda_{\min }(Q)-\mu \lambda_{\max }(P)\right)}
$$

This bound can also be minimized using the conditions (25), together with the condition $\lambda_{\min }(Q)>\mu \lambda_{\max }(P)$.

\section{Estimation of unmodeled dynamics}

Consider now the problem of estimating the states of a fuzzy system in the presence of unmodeled dynamics, i.e., the fuzzy system is of the form

$$
\begin{aligned}
& \dot{\boldsymbol{x}}=\sum_{i=1}^{m} w_{i}(\boldsymbol{z})\left(A_{i} \boldsymbol{x}+B_{i} \boldsymbol{u}+M_{i}\left(A_{\delta i} \boldsymbol{x}+B_{\delta i} \boldsymbol{u}+\theta_{i}\right)\right) \\
& \boldsymbol{y}=C \boldsymbol{x}
\end{aligned}
$$

where $A_{i}, B_{i}, i=1,2, \ldots, m$ are known and the matrices $A_{\delta i}, B_{\delta i}$ and the vectors $\theta_{i}, i=1,2, \ldots, m$ are unknown, but $A_{\delta i}, i=1,2, \ldots, m$ are bounded by a known bound $\mu_{\max }, \max \left\|A_{\delta i}\right\| \leq \mu_{\max }$. This corresponds to the situation when part of the true dynamics is unmodeled. The goal is to determine sufficient conditions and to design an observer that estimates $\boldsymbol{x}$ and also the constant matrices $A_{\delta i}, B_{\delta i}$ and the vector $\theta_{i}, i=1,2, \ldots, m$. Therefore, our goal is to estimate the unknown dynamics.

For the simplicity of the computations, we present only the case when the measurement matrix is common for all rules of the model.

Assumption 1. It is assumed that $M_{i}, i=1,2, \ldots, m$ have full column rank, and $\operatorname{rank}\left(C M_{i}\right)=\operatorname{rank}\left(M_{i}\right), i=1,2, \ldots, m$. 
1) Case 1: Consider first the case when the scheduling vector does not depend on states to be estimated. An observer of the following form is considered:

$$
\begin{aligned}
\dot{\widehat{\boldsymbol{x}}} & =\sum_{i=1}^{m} w_{i}(\boldsymbol{z})\left(A_{i} \widehat{\boldsymbol{x}}+B_{i} \boldsymbol{u}+L_{i}(\boldsymbol{y}-\widehat{\boldsymbol{y}})+M_{i}\left(\widehat{A}_{\delta i} \widehat{\boldsymbol{x}}+\widehat{B}_{\delta i} \boldsymbol{u}+\widehat{\theta}_{i}\right)\right) \\
\widehat{\boldsymbol{y}} & =C \widehat{\boldsymbol{x}} \\
\dot{\widehat{A}}_{\delta i} & =f_{i}\left(\widehat{A}_{\delta i}, \boldsymbol{w}(\boldsymbol{z}), \widehat{\boldsymbol{x}}, \boldsymbol{y}\right) \\
\dot{\widehat{B}}_{\delta i} & =g_{i}\left(\widehat{B}_{\delta i}, \boldsymbol{w}(\boldsymbol{z}), \widehat{\boldsymbol{x}}, \boldsymbol{y}, \boldsymbol{u}\right) \\
\dot{\widehat{\theta}}_{i} & =h_{i}\left(\widehat{\theta}_{i}, \boldsymbol{w}(\boldsymbol{z}), \widehat{\boldsymbol{x}}, \boldsymbol{y}\right)
\end{aligned}
$$

where $L_{i}, i=1,2, \ldots, m$ are the gain matrices for each rule, and the update laws $f_{i}, g_{i}, h_{i}, i=1,2, \ldots, m$ will be determined so that the estimation errors $\boldsymbol{x}-\widehat{\boldsymbol{x}}, A_{\delta i}-\widehat{A}_{\delta i}, B_{\delta i}-\widehat{B}_{\delta i}$, and $\theta_{i}-\widehat{\theta}_{i}$ converge asymptotically to zero.

The error system when using the observer (29) can be expressed as:

$$
\begin{aligned}
\dot{\boldsymbol{e}} & =\sum_{i=1}^{m} w_{i}(\boldsymbol{z})\left[\left(A_{i}-L_{i} C+A_{\delta i}\right) \boldsymbol{e}+M_{i}\left(\bar{A}_{\delta i} \widehat{\boldsymbol{x}}+\bar{B}_{\delta i} \boldsymbol{u}+\bar{\theta}_{i}\right)\right] \\
\boldsymbol{e}_{y} & =C \boldsymbol{e}
\end{aligned}
$$

with $\bar{A}_{\delta i}=A_{\delta i}-\widehat{A}_{\delta i}, \bar{B}_{\delta i}=B_{\delta i}-\widehat{B}_{\delta i}, \bar{\theta}_{i}=\theta_{i}-\widehat{\theta}_{i}$.

Consider first the following part of the error expressed in (30):

$$
\dot{\tilde{\boldsymbol{e}}}=\sum_{i=1}^{m} w_{i}(\boldsymbol{z})\left(A_{i}-L_{i} C+A_{\delta i}\right) \widetilde{\boldsymbol{e}}
$$

Since a bound on $A_{\delta i}, i=1,2, \ldots, m$ is known, i.e., $\max \left\|A_{\delta i}\right\| \leq \mu_{\max }$, stability conditions for perturbed fuzzy systems can be used to render (31) stable and to design the gain matrices $L_{i}$ [42]: find $P=P^{T}>0, Q=Q^{T}>0, L_{i}$, $i=1,2, \ldots, m$ so that

$$
\begin{aligned}
& \mu_{\max } \leq \frac{\lambda_{\min }(Q)}{\lambda_{\max }(P)} \\
& \mathcal{H}\left(P\left(A_{i}-L_{i} C\right)\right) \leq-2 Q
\end{aligned}
$$

Consider now a Lyapunov function of the form

$$
V=\boldsymbol{e}^{T} P \boldsymbol{e}+\sum_{i=1}^{m} \operatorname{tr}\left(\bar{A}_{\delta i}^{T} \bar{A}_{\delta i}\right)+\sum_{i=1}^{m} \operatorname{tr}\left(\bar{B}_{\delta i}^{T} \bar{B}_{\delta i}\right)+\sum_{i=1}^{m}\left(\bar{\theta}_{i}^{T} \bar{\theta}_{i}\right)
$$


for the error system (30), so that $P$ satisfies (32). Then,

$$
\begin{aligned}
\dot{V}= & \sum_{i=1}^{n} w_{i}(\boldsymbol{z}) \boldsymbol{e}^{T}\left[\left(A_{i}-L_{i} C+A_{\delta i}\right)^{T} P+P\left(A_{i}-L_{i} C+A_{\delta i}\right)\right] \boldsymbol{e} \\
& +2 \boldsymbol{e}^{T} P \sum_{i=1}^{m} w_{i}(\boldsymbol{z}) M_{i} \bar{A}_{\delta i} \widehat{\boldsymbol{x}}+2 \boldsymbol{e}^{T} P \sum_{i=1}^{m} w_{i}(\boldsymbol{z}) M_{i} \bar{B}_{\delta i} \boldsymbol{u} \\
& +2 \boldsymbol{e}^{T} P \sum_{i=1}^{m} w_{i}(\boldsymbol{z}) M_{i} \bar{\theta}_{i}-2 \sum_{i=1}^{m} \operatorname{tr}\left(\dot{\widehat{A}}_{\delta i}^{T} \bar{A}_{\delta i}\right)-2 \sum_{i=1}^{m} \operatorname{tr}\left(\dot{\widehat{B}}_{\delta i}^{T} \bar{B}_{\delta i}\right)-2 \sum_{i=1}^{m}\left(\dot{\hat{\theta}}_{i}^{T} \bar{\theta}_{i}\right) \\
= & \sum_{i=1}^{n} w_{i}(\boldsymbol{z}) \boldsymbol{e}^{T} G_{i} \boldsymbol{e}+2 \sum_{i=1}^{m}\left(\operatorname{tr}\left(\widehat{\boldsymbol{x}} \boldsymbol{e}^{T} P M_{i} w_{i}(\boldsymbol{z}) \bar{A}_{\delta i}\right)-\operatorname{tr}\left(\dot{\hat{A}}_{\delta i}^{T} \bar{A}_{\delta i}\right)\right) \\
& +2 \sum_{i=1}^{m}\left(\operatorname{tr}\left(\boldsymbol{u} \boldsymbol{e}^{T} P M_{i} w_{i}(\boldsymbol{z}) \bar{B}_{\delta i}\right)-\operatorname{tr}\left(\dot{\widehat{B}}_{\delta i}^{T} \bar{B}_{\delta i}\right)\right)+2 \sum_{i=1}^{m}\left(\boldsymbol{e}^{T} P M_{i} w_{i}(\boldsymbol{z}) \bar{\theta}_{i}-\dot{\hat{\theta}}_{i}^{T} \bar{\theta}_{i}\right) \\
= & \sum_{i=1}^{n} w_{i}(\boldsymbol{z}) \boldsymbol{e}^{T} G_{i} \boldsymbol{e}+2 \sum_{i=1}^{m} \operatorname{tr}\left(\left(\widehat{\boldsymbol{x}} \boldsymbol{e}^{T} P M_{i} w_{i}(\boldsymbol{z})-\dot{\widehat{A}}_{\delta i}^{T}\right) \bar{A}_{\delta i}\right) \\
& +2 \sum_{i=1}^{m} \operatorname{tr}\left(\left(\boldsymbol{u} \boldsymbol{e}^{T} P M_{i} w_{i}(\boldsymbol{z})-\dot{\widehat{B}}_{\delta i}^{T}\right) \bar{B}_{\delta i}\right)+2 \sum_{i=1}^{m}\left(\boldsymbol{e}^{T} P w_{i}(\boldsymbol{z})-\dot{\hat{\theta}}_{i}^{T}\right) \bar{\theta}_{i}
\end{aligned}
$$

with $G_{i}=\mathcal{H}\left(P\left(A_{i}-L_{i} C+A_{\delta i}\right)\right)$.

Since $V>0$ and from (32) $G_{i}<0$, for $i=1,2, \ldots, m, \dot{V}<0$ is rendered negative definite if $\operatorname{tr}\left(\left(\widehat{\boldsymbol{x}} \boldsymbol{e}^{T} P M_{i} w_{i}(\boldsymbol{z})-\dot{\hat{A}}_{\delta i}^{T}\right) \bar{A}_{\delta i}\right)=0, \operatorname{tr}\left(\left(\boldsymbol{u} \boldsymbol{e}^{T} P M_{i} w_{i}(\boldsymbol{z})-\right.\right.$ $\left.\left.\dot{\hat{B}}_{\delta i}^{T}\right) \bar{B}_{\delta i}\right)$ and $\boldsymbol{e}^{T} P M_{i} w_{i}(\boldsymbol{z})-\dot{\hat{\theta}}_{i}^{T}=0$, for $i=1,2, \ldots, m$. The equations lead to the update laws:

$$
\begin{aligned}
\dot{\widehat{A}}_{\delta i} & =w_{i}(\boldsymbol{z}) M_{i}^{T} P \boldsymbol{e} \widehat{\boldsymbol{x}}^{T} \\
\dot{\widehat{B}}_{\delta i} & =w_{i}(\boldsymbol{z}) M_{i}^{T} P \boldsymbol{e} \boldsymbol{u}^{T} \\
\dot{\widehat{\theta}}_{i} & =w_{i}(\boldsymbol{z}) M_{i}^{T} P \boldsymbol{e}
\end{aligned}
$$

Note, that in general $\boldsymbol{e}$ is not directly available. However, given Assumption 1, there exist matrices $\Lambda_{i}, i=1,2, \ldots, m$ so that $\Lambda_{i} C=M_{i}^{T} P: \Lambda_{i}=M_{i}^{T} P C^{\dagger}$, where $C^{\dagger}$ denotes the Moore-Penrose pseudoinverse of $C$.

Therefore, the update laws can be expressed as:

$$
\begin{aligned}
\dot{\hat{A}}_{\delta i} & =w_{i}(\boldsymbol{z}) M_{i}^{T} P C^{\dagger} \boldsymbol{e}_{y} \widehat{\boldsymbol{x}}^{T} \\
\dot{\hat{B}}_{\delta i} & =w_{i}(\boldsymbol{z}) M_{i}^{T} P C^{\dagger} \boldsymbol{e}_{y} \boldsymbol{u}^{T} \\
\dot{\hat{\theta}}_{i} & =w_{i}(\boldsymbol{z}) M_{i}^{T} P C^{\dagger} \boldsymbol{e}_{y}
\end{aligned}
$$


If all the rules are sufficiently excited, both the error system and the estimation error of the unknown matrices are asymptotically stable. It can easily be seen that, assuming nonzero and varying $\boldsymbol{x}, \boldsymbol{u}$, the only invariant set of the error system (30) is $\boldsymbol{e}=0, \bar{A}_{\delta i}=0, \bar{B}_{\delta i}=0$ and $\bar{\theta}_{i}=0$. If $w_{i}(\boldsymbol{z}), i=1,2, \ldots, m$ are sufficiently smooth and the fuzzy model is defined on a compact set of variables, then based on Barbalat's lemma and LaSalle's invariance principle, the dynamics (30), together with the update laws above are asymptotically stable.

The results can be summarized as follows:

Theorem 8. The error dynamics (30) is asymptotically stable, if the update laws (34) are used and, furthermore, there exist $P=P^{T}>0, Q=Q^{T}>0, L_{i}$, $i=1,2, \ldots, m$ so that

$$
\begin{aligned}
& P>0 \\
& \mathcal{H}\left(P\left(A_{i}-L_{i} C\right)\right)<-2 Q \quad i=1,2, \ldots, m \\
& \mu_{\max } \leq \frac{\lambda_{\min }(Q)}{\lambda_{\max }(P)}
\end{aligned}
$$

2) Case 2: Consider now the case when $\boldsymbol{z}$ depends on $\widehat{\boldsymbol{x}}$. The error system (30) becomes

$$
\begin{aligned}
\dot{\boldsymbol{e}} & =\sum_{i=1}^{m} w_{i}(\widehat{\boldsymbol{z}})\left[\left(A_{i}-L_{i} C+A_{\delta i}\right) \boldsymbol{e}+M_{i}\left(\bar{A}_{\delta i} \widehat{\boldsymbol{x}}+\bar{B}_{\delta i} \boldsymbol{u}+\bar{\theta}_{i}\right)\right] \\
& +\sum_{i=1}^{m}\left(w_{i}(\boldsymbol{z})-w_{i}(\widehat{\boldsymbol{z}})\right) \cdot\left(A_{i} \boldsymbol{x}+B_{i} \boldsymbol{u}+M_{i}\left(A_{\delta i} \boldsymbol{x}+B_{\delta i} \boldsymbol{u}+\theta_{i}\right)\right) \\
\boldsymbol{e}_{y} & =C \boldsymbol{e}
\end{aligned}
$$

Under the assumption that $\| \sum_{i=1}^{m}\left(w_{i}(\boldsymbol{z})-w_{i}(\widehat{\boldsymbol{z}})\right)\left(A_{i} \boldsymbol{x}+B_{i} \boldsymbol{u}+M_{i}\left(A_{\delta i} \boldsymbol{x}+\right.\right.$ $\left.\left.B_{\delta i} \boldsymbol{u}+\theta_{i}\right)\right)\|\leq \mu\| \boldsymbol{e} \|$, combining the conditions in Theorems 5 and 8 , we get:

Corollary 2. The error system (36), together with the update laws

$$
\begin{aligned}
\dot{\widehat{A}}_{\delta i} & =w_{i}(\widehat{\boldsymbol{z}}) M_{i}^{T} P C^{\dagger} \boldsymbol{e}_{y} \widehat{\boldsymbol{x}}^{T} \\
\dot{\widehat{B}}_{\delta i} & =w_{i}(\widehat{\boldsymbol{z}}) M_{i}^{T} P C^{\dagger} \boldsymbol{e}_{y} \boldsymbol{u}^{T} \\
\dot{\hat{\theta}}_{i} & =w_{i}(\widehat{\boldsymbol{z}}) M_{i}^{T} P C^{\dagger} \boldsymbol{e}_{y}
\end{aligned}
$$


is asymptotically stable, if there exist $P=P^{T}>0, Q=Q^{T}>0, L_{i}, i=$ $1,2, \ldots, m$ so that

$$
\begin{aligned}
& P>0 \\
& \mathcal{H}\left(P\left(A_{i}-L_{i} C\right)\right)<-Q \quad i=1,2, \ldots, m \\
& \sum_{i=1}^{m}\left\|\left(w_{i}(\boldsymbol{z})-w_{i}(\widehat{\boldsymbol{z}})\right) \cdot\left(A_{i} \boldsymbol{x}+B_{i} \boldsymbol{u}+M_{i}\left(A_{\delta i} \boldsymbol{x}+B_{\delta i} \boldsymbol{u}+\theta_{i}\right)\right)\right\| \leq \mu\|\boldsymbol{e}\| \\
& \left(\begin{array}{cr}
Q-\mu^{2} I & P \\
P & I
\end{array}\right)>0
\end{aligned}
$$

The proof follows directly.

Remark: Note, that if the measurement matrix is different for each rule of the fuzzy model, the update laws for the matrices of the unknown dynamics can still be expressed as (33). Update laws similar to (34) can be derived if there exist $\left(\sum_{i=1}^{m} w_{i}(\boldsymbol{z}) C_{i}\right)^{\dagger}, \forall \boldsymbol{z}$ (Case 1), and $\left(\sum_{i=1}^{m} w_{i}(\widehat{\boldsymbol{z}}) C_{i}\right)^{\dagger}, \forall \widehat{\boldsymbol{z}}$ (Case 2), $i=1,2, \ldots, m$. In this case, the update laws are

$$
\begin{aligned}
\dot{\widehat{A}}_{\delta i} & =w_{i}(\boldsymbol{z}) M_{i}^{T} P\left(\sum_{i=1}^{m} w_{i}(\boldsymbol{z}) C_{i}\right)^{\dagger} \boldsymbol{e}_{y} \widehat{\boldsymbol{x}}^{T} \\
\dot{\widehat{B}}_{\delta i} & =w_{i}(\boldsymbol{z}) M_{i}^{T} P\left(\sum_{i=1}^{m} w_{i}(\boldsymbol{z}) C_{i}\right)^{\dagger} \boldsymbol{e}_{y} \boldsymbol{u}^{T} \\
\dot{\widehat{\theta}}_{i} & =w_{i}(\boldsymbol{z}) M_{i}^{T} P\left(\sum_{i=1}^{m} w_{i}(\boldsymbol{z}) C_{i}\right)^{\dagger} \boldsymbol{e}_{y}
\end{aligned}
$$

if the scheduling vector does not depend on the states to be estimated and

$$
\begin{aligned}
\dot{\widehat{A}}_{\delta i} & =w_{i}(\widehat{\boldsymbol{z}}) M_{i}^{T} P\left(\sum_{i=1}^{m} w_{i}(\widehat{\boldsymbol{z}}) C_{i}\right)^{\dagger} \boldsymbol{e}_{y} \widehat{\boldsymbol{x}}^{T} \\
\dot{\widehat{B}}_{\delta i} & =w_{i}(\widehat{\boldsymbol{z}}) M_{i}^{T} P\left(\sum_{i=1}^{m} w_{i}(\widehat{\boldsymbol{z}}) C_{i}\right)^{\dagger} \boldsymbol{e}_{y} \boldsymbol{u}^{T} \\
\dot{\widehat{\theta}}_{i} & =w_{i}(\widehat{\boldsymbol{z}}) M_{i}^{T} P\left(\sum_{i=1}^{m} w_{i}(\widehat{\boldsymbol{z}}) C_{i}\right)^{\dagger} \boldsymbol{e}_{y}
\end{aligned}
$$

if the scheduling vector depends on $\widehat{\boldsymbol{x}}$ and the observer gains are given by (35) and (38), respectively. Note that, for this case, to have a unique solution, 
Assumption 1 has to be modified to $\operatorname{rank}\left(\sum_{j=1}^{m} w_{j}(\boldsymbol{z}) C_{j} M_{i}\right)=\operatorname{rank}\left(M_{i}\right), i=$ $1,2, \ldots, m, \forall \boldsymbol{z}$.

Remark: The results can also be applied if the unknown matrices have slow dynamics, such that $\dot{A_{\delta i}} \simeq 0$, etc.

\section{Related work}

Our objective is to estimate the states and unknown inputs of a nonlinear system. In this paper we consider nonlinear systems represented by TS fuzzy models, and unknown inputs that are polynomial functions of time or can be approximated by polynomials in time. Note that our goal is to estimate the states of the given physically relevant model, and not a transformation of the states. To our knowledge, no other work in the literature pertains to this class of problems. Many existing adaptive observers [11-16, 23, 47-49] require that the system is or can be transformed into the observer canonical form. By assuming a canonical form, the physical meaning of the state variables is lost.

In fault detection and isolation, observer based methods have widely been used (see [50] and the references therein). However, these methods usually concern linear systems [51] and they do not estimate the faults. A method for TS systems in descriptor form has been proposed in [39] to estimate the states in the presence of unknown inputs. This method is based either on decoupling the unknown inputs, or on attenuating their effects on the states. If decoupling is possible, the states are correctly estimated, and single faults can be isolated by using a bank of observers. However, the faults (unknown inputs) cannot be reconstructed. For the case when the decoupling of all the unknown inputs is not possible, the authors of [39] also proposed a method to attenuate their effect on the states. However, the estimation error is proportional with the norm of the unknown inputs. In the current paper we consider unknown inputs that are polynomial in time, whose norm generally grows unbounded and therefore, the estimation error grows unlimited if the attenuating method of [39] is used.

Several methods $[31,32,48]$ exist for nonlinear systems composed of a known 
linear part and a Lipschitz nonlinearity affected by unknown inputs. An estimation method has been provided in [26] for a more general class of nonlinear systems, where the known part of the nonlinearity is Lipschitz in the states and inputs. However, this method relies on an assumption (Assumption 2 in [26]) related to a rank condition on a matrix composed of the direct feedthrough term and of a distribution matrix of the unknown nonlinear terms affected by the unknown inputs. Since in this paper we do not consider unknown inputs that affect the measurements directly, this assumption is generally not satisfied, and therefore the method of [26] cannot estimate the unknown inputs.

For SISO LTI systems, the adaptive observer design has been largely investigated (see [10] and the references therein). However, when applying adaptive state and input observers designed for linear systems, such as the approach proposed in [27] for a nonlinear system, the observer can only be used in a small neighborhood of the linearization point. Therefore, such observers are rarely able to estimate the states and the inputs of the nonlinear system. Moreover, they can only deal with constant or slowly varying inputs.

\section{Example}

We illustrate the proposed design method on a simulation example. The system under consideration is the dynamic model of a missile, adopted from [52], illustrated in Figure 1. The nonlinear state-space equations are:

$$
\begin{aligned}
\dot{\alpha} & =K_{\alpha} M C_{n}(\alpha, \delta, M) \cos (\alpha)+q \\
\dot{q} & =K_{q} M^{2} C_{m}(\alpha, \delta, M)
\end{aligned}
$$

The plant variables are given in Table 1, and the parameters in Table 2.

The Mach number $M$ is an exogenous scheduling variable. The angle of attack $\alpha$ and the pitch rate $q$ are measured. The actual tail fin deflection and the unknown inputs acting on the system have to be estimated. The aerodynamic 


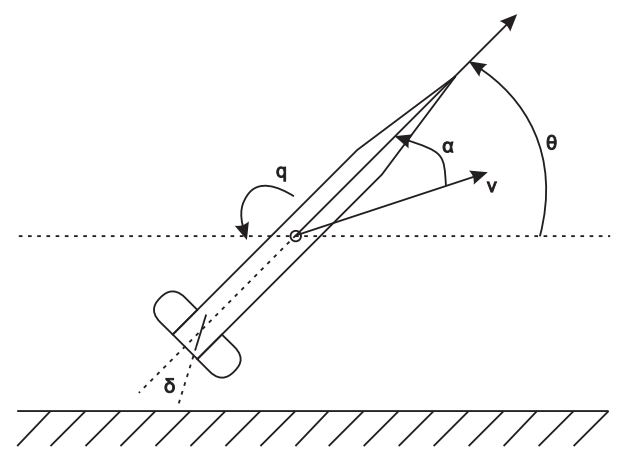

Figure 1: Schematic representation of a missile.

Table 1: Plant variables.

$\alpha \quad$ Angle of attack [deg]

$q \quad$ Pitch rate $[\mathrm{deg} / \mathrm{s}]$

$M \quad$ Mach number

$\delta_{c} \quad$ Commanded tail fin deflection [deg]

$\delta \quad$ Actual tail fin deflection [deg]

coefficients are expressed as:

$$
\begin{aligned}
& C_{n}(\alpha, \delta, M)=\operatorname{sgn}(\alpha)\left[a_{n}|\alpha|^{3}+b_{n}|\alpha|^{2}+c_{n}(2-M / 3)|\alpha|\right]+d_{n} \delta \\
& C_{m}(\alpha, \delta, M)=\operatorname{sgn}(\alpha)\left[a_{m}|\alpha|^{3}+b_{m}|\alpha|^{2}+c_{m}(-7+8 M / 3)|\alpha|\right]+d_{m} \delta
\end{aligned}
$$

The dynamics of the tail fin actuator are described by a first-order linear model:

$$
\dot{\delta}=\omega_{a} \delta+\omega_{a} \delta_{c}
$$

In order to use the proposed design, first an approximate TS fuzzy model of the system (41) is constructed. For the Mach number $M$, five points $M \in$ $\{2,2.5,3,3.5,4\}$ and for the angle of attack seven points $\alpha \in\{-15,-10,-5,0,5,10,15\}$ are chosen as centers of the membership functions. The membership functions for $M$ are depicted in Figure 2. The scheduling vector consists of the Mach number $M$ and the angle of attack $\alpha$, which is also a state to be estimated. An example of a rule is: 
Table 2: Plant parameters.

\begin{tabular}{ll}
\hline$P_{0}=973.3$ & Static pressure at 20000 feet $\left[\mathrm{lbs} / \mathrm{ft}^{2}\right]$ \\
$S=0.44$ & Surface area $\left[\mathrm{ft}^{2}\right]$ \\
$m=13.98$ & Mass[slugs $]$ \\
$v_{s}=1036.4$ & Speed of sound at $20000 \mathrm{feet}[\mathrm{ft} / \mathrm{s}]$ \\
$d=0.75$ & Diameter $[\mathrm{ft}]$ \\
$I_{y}=182.5$ & Pitch moment of inertia $\left[\mathrm{slug} \mathrm{ft}^{2}\right]$ \\
$\omega_{a}=225$ & \\
$K_{\alpha}=(\pi / 180) 0.7 P_{0} S /\left(m v_{s}\right)$ & \\
$K_{q}=(\pi / 180) 0.7 P_{0} S d / I_{y}$ & \\
$a_{n}=0.000103\left[\mathrm{deg}^{-3}\right]$ & $a_{m}=0.000215\left[\mathrm{deg}^{-3}\right]$ \\
$b_{n}=-0.00945\left[\mathrm{deg}^{-2}\right]$ & $b_{m}=-0.0195\left[\mathrm{deg}^{-2}\right]$ \\
$c_{n}=-0.1696\left[\mathrm{deg}^{-1}\right]$ & $c_{m}=-0.051\left[\mathrm{deg}^{-1}\right]$ \\
$d_{n}=-0.034\left[\mathrm{deg}^{-1}\right]$ & $d_{m}=-0.206\left[\mathrm{deg}^{-1}\right]$ \\
\hline
\end{tabular}

If $M$ is approximately 3 and $\alpha$ is approximately 5 then $\dot{\boldsymbol{x}}_{i}=A \boldsymbol{x}+B u+a$, with

$$
\begin{aligned}
A & =\left(\begin{array}{ccc}
-0.0012 & 1.0 & 0.0 \\
-0.0445 & 0 & -0.0399 \\
0 & 0 & -225.0
\end{array}\right) \\
B & =\left(\begin{array}{lll}
0 & 0 & 225
\end{array}\right)^{T} \\
a & =\left(\begin{array}{lll}
0.0056 & 0.0839 & 0
\end{array}\right)^{T}
\end{aligned}
$$

where $\boldsymbol{x}=\left[\begin{array}{lll}\alpha & q & \delta\end{array}\right]^{T}$.

The affine consequent models in the fuzzy rules are obtained by Taylor series expansion around each combination of the chosen $M$ and $\alpha$. To compute the membership degree of the scheduling vector $\boldsymbol{z}=[M \alpha]^{T}$, the algebraic product operator is used.

To simulate the system, the input was randomly generated. For the considered estimations (unmodeled dynamics and unknown input, respectively), the initial conditions for the nonlinear system were $\left[\begin{array}{lll}15 & 2.3 & 3\end{array}\right]^{T}$, while the initial 


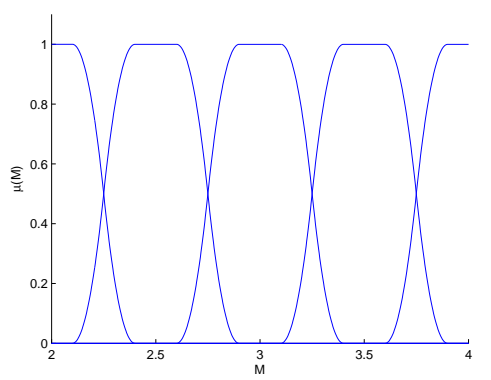

Figure 2: Membership functions for the Mach number M.

conditions used for the states and parameters to be estimated were zero. The LMIs for designing the observer in each case were solved using the Yalmip toolbox [53]. The unknown input/unmodelled dynamics is assumed to affect $\alpha$ and $q$, therefore $M_{i}=\left(\begin{array}{ll}1 & 0 \\ 0 & 1 \\ 0 & 0\end{array}\right), i=1,2, \ldots, m$.

1) Adapting the TS model: Since the consequents in the fuzzy rules were obtained by linearizing the nonlinear model, the local models represent the nonlinear system exactly only in the linearization points, i.e., in the combinations of the points chosen for $M$ and $\alpha$. When the system is not close to the linearization point or more than one membership function is activated, the accuracy of the TS model decreases. This can be seen as if part of the actual dynamics were unmodelled. In order to obtain a better approximation of the nonlinear system, first an observer to estimate this "unmodeled dynamics" was designed, according to Theorem 8 . This observer estimates the states and updates the system matrices according to equation (34). The estimation error for the states is presented in Figure 3. As can be seen, the estimated states converge to the states of the nonlinear system. As a result of using this observer, for instance the rule corresponding to the approximation in $M=3$ and $\alpha=5$ has been changed to:

If $M$ is approximately 3 and $\alpha$ is approximately 5 then $\dot{\boldsymbol{x}}_{i}=A \boldsymbol{x}+B u+a$, 
with

$$
\begin{aligned}
A & =\left(\begin{array}{ccc}
0.0496 & 1.1488 & 0.0062 \\
-0.0164 & -0.0179 & -0.0402 \\
0 & 0 & -225.0
\end{array}\right) \\
B & =\left(\begin{array}{ccc}
-0.0032 & -0.0003 & 225.0
\end{array}\right)^{T} \\
a & =\left(\begin{array}{lll}
0.00475 & -0.0031 & 0
\end{array}\right)^{T}
\end{aligned}
$$

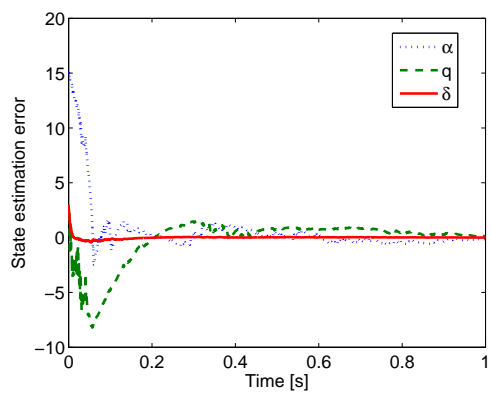

Figure 3: Estimation of unmodeled dynamics: state estimation error.

2) Unknown input estimation: Now assume that an unknown input $\boldsymbol{d}$ is acting on $\alpha$ and $q$. Three cases are considered: 1) constant input, 2) secondorder polynomial input, and 3) a non-polynomial input. The application of the proposed approach and the obtained results are detailed in what follows.

1. In the first case, $\boldsymbol{d}$ is constant, $\boldsymbol{d}=\left[\begin{array}{ll}5 & 10\end{array}\right]^{T}$. The observer is designed based on the conditions of Theorem 6 , with the first derivative of the unknown input being already zero, i.e., correctly assuming a bias in the model. The state estimation error is presented in Figure 4(a). The unknown input and its estimate is presented in Figure 4(b). Both the estimated states and input converge to the correct ones, as expected.

To compare with another method, we have implemented ${ }^{1}$ the approach

\footnotetext{
${ }^{1}$ We have also implemented the method described in [26]. However, due to the fact that Assumption 2 in [26] is not satisfied, only the states are correctly estimated and not the unknown inputs. Therefore, those results are not presented here.
} 
described in [27]. The state estimation error and the estimated inputs are presented in Figures 4(c) and 4(d), respectively. As can be seen, with the approach proposed in this paper, the estimated states and inputs converge to their true values faster.
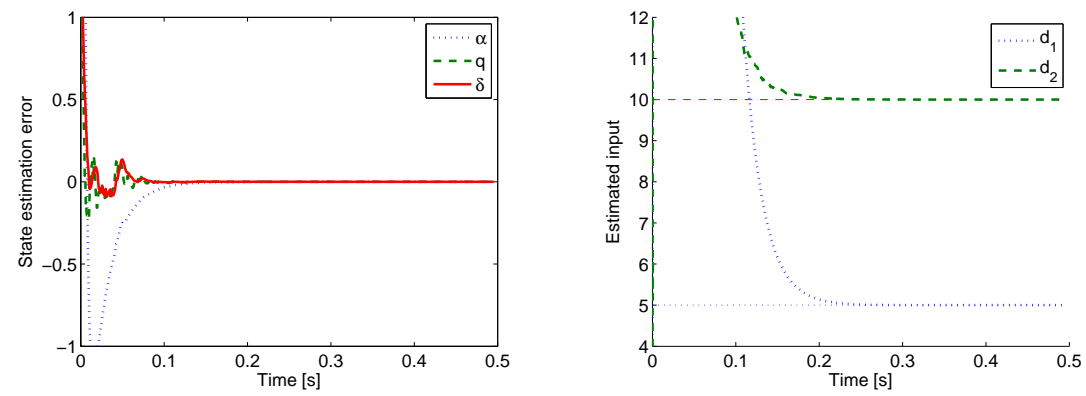

(a) Estimation error for the states using the (b) The unknown input (thin line) and its proposed approach. estimate (thick line) using the proposed approach.
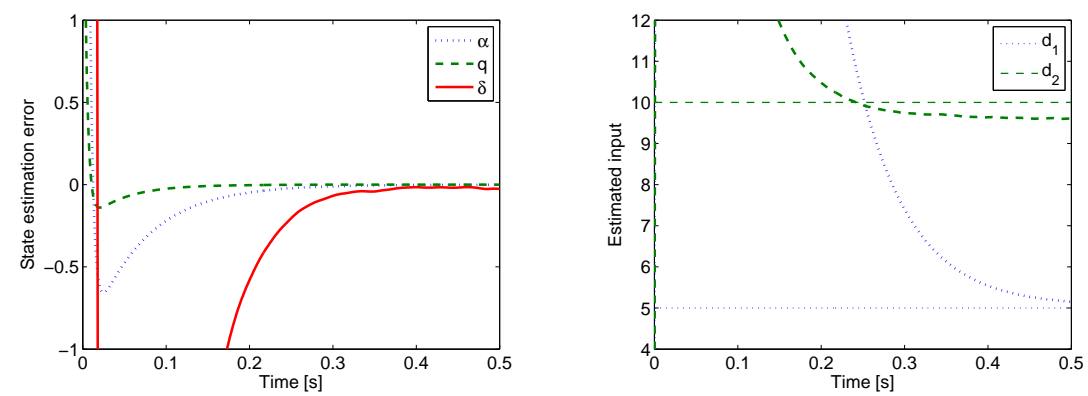

(c) Estimation error for the states using the (d) The unknown input (thin line) and its approach in [27]. estimate (thick line) using the approach in $[27]$.

Figure 4: Estimation results for constant input.

2. In the second case, $\boldsymbol{d}$ is a second order polynomial in time, $\boldsymbol{d}=\left[\begin{array}{ll}5 & 10\end{array}\right]^{T} t^{2}+$ $\left[\begin{array}{ll}3 & 1\end{array}\right]^{T} t+\left[\begin{array}{ll}2 & 4\end{array}\right]^{T}$. The observer is designed in the same manner as for the $\boldsymbol{d}$ in the constant input case, but using three derivatives in Theorem 6 . The estimation error for the states is presented in Figure 5(a) and the unknown inputs and their estimates in Figure 5(b). The estimated states 
and inputs converge to the correct states and inputs as expected.

The state estimation error and the estimated inputs using the approach in [27] are presented in Figures 5(c) and 5(d), respectively. Similarly to the previous case, using the proposed approach, the estimated states and inputs converge faster to their true values.
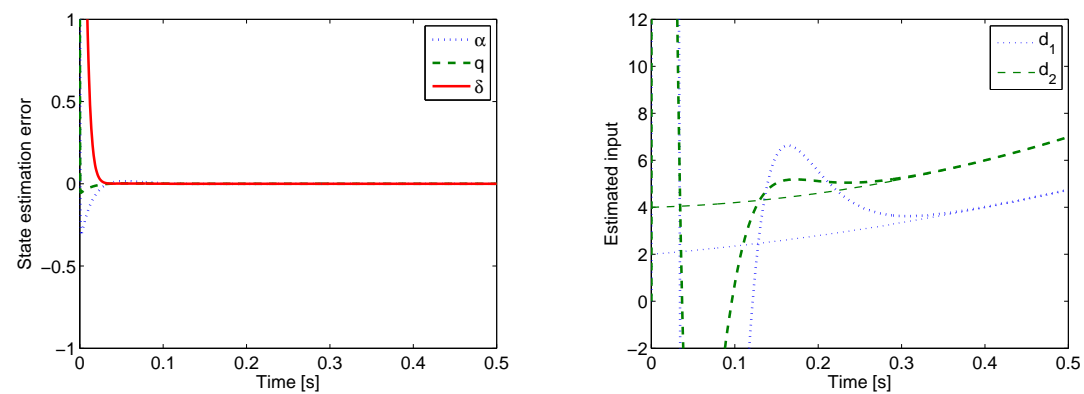

(a) Estimation error for the states using the (b) The unknown input (thin line) and its proposed approach. estimate (thick line) using the proposed approach.
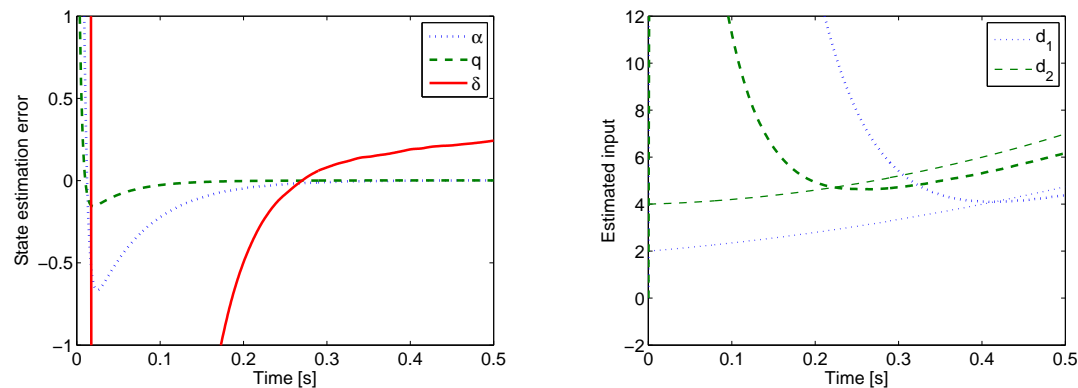

(c) Estimation error for the states using the (d) The unknown input (thin line) and its approach in [27]. estimate (thick line) using the approach in [27].

Figure 5: Estimation results for second order input.

3. The last case considered is with the true unknown input $\boldsymbol{d}$ acting on the system being non-polynomial, given by $\boldsymbol{d}=\left[\begin{array}{ll}1 & 3\end{array}\right]^{T} \sin (t)+\left[\begin{array}{ll}4 & 2\end{array}\right]^{T} \cos (t)$, but the observer is computed by (incorrectly) assuming a linear input, i.e., a ramp. The estimation error for the states is presented in Figure 6(a) 
and the unknown inputs and their estimate in Figure 6(b). The estimated input does not converge exponentially to the exact value of the true input (see Figure 7), but only to a ball around it. The ultimate bound on the error (state and input), computed for this observer is $38.6 \cdot\|\boldsymbol{d}\| / \sigma$ with $\|\boldsymbol{d}\|$ the norm of the input and $\sigma$ arbitrarily chosen in $(0,1)$. However, based on the estimation errors presented in Figures 6(a) and 7, one can conclude that the computed bound is rather conservative.
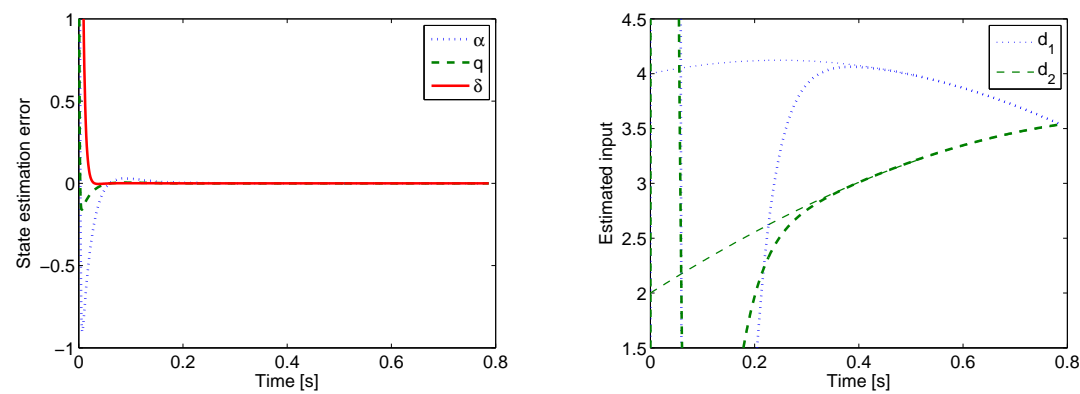

(a) Estimation error for the states using the (b) The unknown input (thin line) and its proposed approach. estimate (thick line) using the proposed approach.
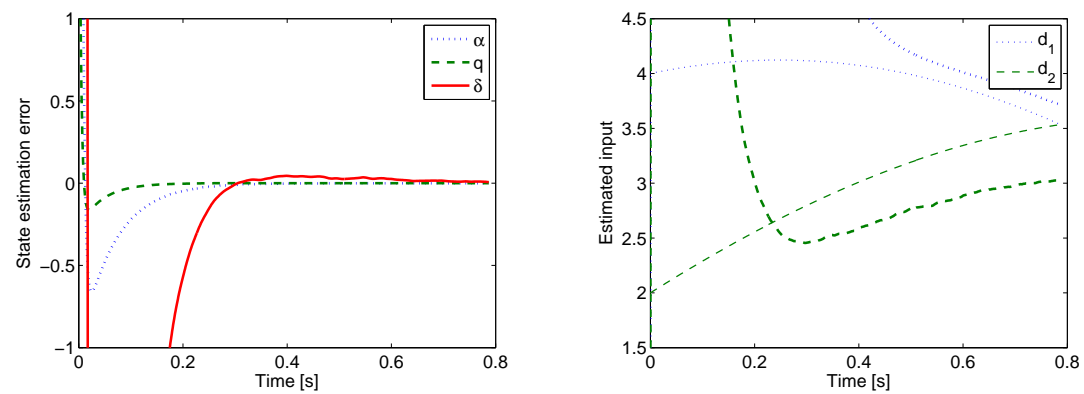

(c) Estimation error for the states using the (d) The unknown input (thin line) and its approach in [27]. estimate (thick line) using the approach in $[27]$.

Figure 6: Estimation results for non-polynomial input.

The state estimation error and the estimated inputs using the approach in [27] for this case also are presented in Figures 6(c) and 6(d), respectively. 


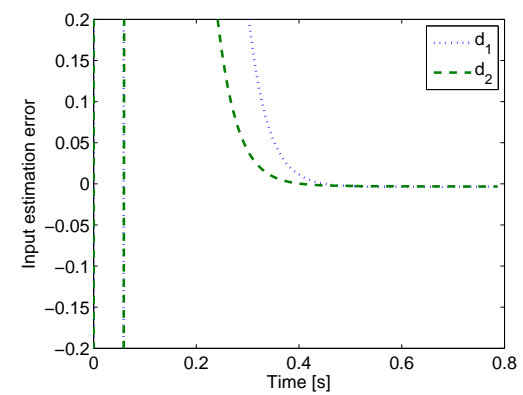

Figure 7: Input estimation error using the proposed approach.

As in the previous cases, using the approach proposed in this paper, the estimates converge faster to their true values.

\section{Conclusions}

In this paper a method was proposed for estimating the state and unknown inputs of TS fuzzy systems. The design of unknown input observers is important in practice, since in many cases not all the inputs are known. These unknown inputs can represent disturbances acting on the process, effects of unmodeled dynamics, or actuator failures. The proposed observer was designed based on the known part of the fuzzy model. Sufficient conditions were given for the stability of the observer and the computation of the observer gains is based on solving a system of LMIs. Conditions on the exponential convergence of the observer in the case of polynomial unknown inputs were presented and the design guarantees an ultimate bound on the error signal. In the case of estimating unmodeled dynamics, sufficient conditions were given for the asymptotic convergence of the observer. The design methods were illustrated on an example of a missile. The simulation results showed that the proposed observer is able to estimate both the states and inputs simultaneously.

In our future research, we will investigate the extension of the proposed method to stable state-feedback controller design and also adaptive observerbased stable control design for practical control applications. Two main chal- 
lenges that have to be solved in this context are: the controller has to compensate for the unknown input that is time-varying; moreover, if the scheduling vector depends on states that have to be estimated, the separation principle does not hold. We will also investigate conditions under which fuzzy observers can be used to estimate other than polynomial types of unknown inputs.

\section{Acknowledgment}

This research is sponsored by Senter, Ministry of Economic Affairs of the Netherlands within the project Interactive Collaborative Information Systems (grant no. BSIK03024), and by the International Campus on Safety and Intermodality in Transportation the Nord-Pas-de-Calais Region, the European Community, the Regional Delegation for Research and Technology, the French Ministry of Higher Education and Research, and the French National Center for Scientific Research.

\section{References}

[1] T. Takagi, M. Sugeno, Fuzzy identification of systems and its applications to modeling and control, IEEE Transactions on Systems, Man, and Cybernetics 15 (1) (1985) 116-132.

[2] C. Fantuzzi, R. Rovatti, On the approximation capabilities of the homogeneous Takagi-Sugeno model, in: Proceedings of the Fifth IEEE International Conference on Fuzzy Systems, New Orleans, LA, 1996, pp. 10671072 .

[3] K. Tanaka, T. Ikeda, H. Wang, Fuzzy regulators and fuzzy observers: relaxed stability conditions and LMI-based designs, IEEE Transactions on Fuzzy Systems 6 (2) (1998) 250-265.

[4] M. Johansson, A. Rantzer, K. Arzen, Piecewise quadratic stability of fuzzy systems, IEEE Transactions on Fuzzy Systems 7 (6) (1999) 713-722. 
[5] P. Bergsten, R. Palm, D. Driankov, Fuzzy observers, in: Proceedings of the 10th IEEE International Conference on Fuzzy Systems, Vol. 2, Melbourne, Australia, 2001, pp. 700-703.

[6] G. Feng, A survey on analysis and design of model-based fuzzy control systems, IEEE Transactions on Fuzzy Systems 14 (5) (2006) 676-697.

[7] K. Tanaka, H. Wang, Fuzzy regulators and fuzzy observers: a linear matrix inequality approach, in: Proceedings of the 36th IEEE Conference on Decision and Control, Vol. 2, San Diego, California, 1997, pp. 1315-1320.

[8] P. Bergsten, R. Palm, D. Driankov, Observers for Takagi-Sugeno fuzzy systems, IEEE Transactions on Systems, Man and Cybernetics, Part B 32 (1) (2002) 114-121.

[9] R. Palm, P. Bergsten, Sliding mode observer for a Takagi-Sugeno fuzzy system, in: Proceedings of the 9th IEEE International Conference on Fuzzy Systems, Vol. 2, San Antonio, Texas, 2000, pp. 665-670.

[10] K. S. Narendra, A. M. Annaswamy, Stable Adaptive Systems, PrenticeHall, Inc., Upper Saddle River, New Jersey, USA, 1989.

[11] J.-H. Park, G.-T. Park, Adaptive fuzzy observer with minimal dynamic order for uncertain nonlinear systems, IEE Proceedings-Control Theory and Applications 150 (2) (2003) 189-197.

[12] S. Tong, H.-X. Li, W. Wang, Observer-based adaptive fuzzy control for SISO nonlinear systems, Fuzzy Sets and Systems 148 (3) (2004) 355-376.

[13] J.-H. Park, G.-T. Park, S.-H. Kim, C.-J. Moon, Output-feedback control of uncertain nonlinear systems using a self-structuring adaptive fuzzy observer, Fuzzy Sets and Systems 151 (1) (2005) 21-42.

[14] H. Ho, Y. Wong, A. Rad, W. Lo, State observer based indirect adaptive fuzzy tracking control, Simulation Modelling Practice and Theory 7 (13) (2005) 646-663. 
[15] Y. Wang, T. Chai, Output-feedback control of uncertain nonlinear systems using adaptive fuzzy observer, in: Proceedings of the American Control Conference, Vol. 4, Portland, Oregon, 2005, pp. 2613-2618.

[16] J.-H. Park, P.-S. Yoon, G.-T. Park, Robust adaptive observer using fuzzy systems for uncertain nonlinear systems, in: Proceedings of the 10th IEEE International Conference on Fuzzy Systems, Vol. 2, Melbourne, Australia, 2001, pp. 749-752.

[17] S. Labiod, T. M. Guerra, Adaptive fuzzy control of a class of SISO nonaffine nonlinear systems, Fuzzy Sets and Systems 158 (10) (2007) 1126-1137.

[18] S. Tong, W. Wang, L. Qu, Decentralized robust control for uncertain T-S fuzzy large-scale systems with time-delay, International Journal of Innovative Computing, Information and Control 3 (3) (2007) 657-672.

[19] S. Tong, H.-H. Li, Observer-based robust fuzzy control of nonlinear systems with parametric uncertainties, Fuzzy Sets and Systems 131 (2) (2002) 165184.

[20] M. Chadli, A. El Hajjaji, Comment on "Observer-based robust fuzzy control of nonlinear systems with parametric uncertainties", Fuzzy Sets and Systems 157 (9) (2006) 1276-1281.

[21] Q. Zhang, A. Xu, Implicit adaptive observers for a class of nonlinear systems, in: Proceedings of the American Control Conference, Vol. 2, Arlington, Virginia, 2001, pp. 1551-1556.

[22] Y. Zhu, P. Pagilla, Adaptive controller and observer design for a class of nonlinear systems, in: Proceedings of the 42nd IEEE Conference on Decision and Control, Vol. 2, Maui, Hawaii, 2003, pp. 1687-1692.

[23] W.-J. Wang, L. Luoh, Stability and stabilization of fuzzy large-scale systems, IEEE Transactions on Fuzzy Systems 12 (3) (2004) 309-315. 
[24] J. Ruiz Vargas, E. Hemerly, Adaptive observers for unknown general nonlinear systems, IEEE Transactions on Systems, Man and Cybernetics, Part B 31 (5) (2001) 683-690.

[25] N. Hovakimyan, A. Calise, V. Madyastha, An adaptive observer design methodology for bounded nonlinear processes, in: Proceedings of the 41st IEEE Conference on Decision and Control, Vol. 4, Las Vegas, Nevada, 2002, pp. $4700-4705$.

[26] Q. P. Ha, H. Trinh, State and input simultaneous estimation for a class of nonlinear systems, Automatica 40 (10) (2004) 1779-1785.

[27] Y. Xiong, M. Saif, Unknown disturbance inputs estimation based on a state functional observer design, Automatica 39 (8) (2003) 1389-1398.

[28] S. Zhou, J. Lam, A. Xue, $H_{\infty}$ filtering of discrete-time fuzzy systems via basis-dependent Lyapunov function approach, Fuzzy Sets and Systems 158 (2) (2007) 180-193.

[29] C.-S. Tseng, B.-S. Chen, $H_{\infty}$ decentralized fuzzy model reference tracking control design for nonlinear interconnected systems, IEEE Transactions on Fuzzy Systems 9 (6) (2001) 795-809.

[30] C.-S. Tseng, B.-S. Chen, $H_{\infty}$ fuzzy estimation for a class of nonlinear discrete-time dynamic systems, IEEE Transactions on Signal Processing 49 (11) (2001) 2605-2619.

[31] A. Pertew, H. Marquez, Q. Zhao, $H_{\infty}$ synthesis of unknown input observers for non-linear Lipschitz systems, International Journal of Control 78 (15) (2005) 1155-1165.

[32] A. Pertew, H. Marquez, Q. Zhao, $H_{\infty}$ observer design for Lipschitz nonlinear systems, IEEE Transactions on Automatic Control 51 (7) (2006) $1211-1216$. 
[33] M. Corless, J. Tu, State and input estimation for a class of uncertain systems, Automatica 34 (6) (1998) 757-764.

[34] A. L. Sheldrake, Estimation of Plant Electrical Load, John Wiley and Sons, 2005, Ch. 1, pp. 1-18.

[35] D. D. Li, C. Chen, A novel approach to estimate load factor of variablespeed wind turbines, IEEE Transactions on Power Systems 20 (2) (2005) $1186-1188$.

[36] K. Guelton, S. Delprat, T.-M. Guerra, An alternative to inverse dynamics joint torques estimation in human stance based on a Takagi-Sugeno unknown-inputs observer in the descriptor form, Control Engineering Practice 16 (12) (2008) 1414-1426.

[37] Y. Wang, M. Papageorgiou, Real-time freeway traffic state estimation based on extended Kalman filter: A general approach, Transportation Research B 39 (2) (2005) 141-167.

[38] T. L. Liao, N. S. Huang, An observer based approach for chaotic synchronization with applications to secure communications, IEEE Transactions on Circuits and Systems 46 (9) (1999) 1144-1150.

[39] B. Marx, D. Koenig, J. Ragot, Design of observers for Takagi-Sugeno descriptor systems with unknown inputs and application to fault diagnosis, IET Control Theory \& Applications 1 (5) (2007) 1487-1495.

[40] B. Kulcsar, J. Bokor, J. Shinar, Unknown input reconstruction for LPV systems, International Journal of Robust and Nonlinear Control 20 (5) (2009) 579-595.

[41] H. K. Khalil, Nonlinear Systems, Prentice-Hall, Upper Saddle River, New Jersey, USA, 2002.

[42] P. Bergsten, Observers and controllers for Takagi-Sugeno fuzzy systems, Ph.D. thesis, Örebro University, Sweden (2001). 
[43] H. Tuan, P. Apkarian, T. Narikiyo, Y. Yamamoto, Parameterized linear matrix inequality techniques in fuzzy control system design, IEEE Transactions on Fuzzy Systems 9 (2) (2001) 324-332.

[44] M. Johansson, A. Rantzer, Computation of piecewise quadratic Lyapunov functions for hybrid systems, IEEE Transactions on Automatic Control 43 (4) (1998) 555-559.

[45] M. Johansson, Piecewise linear control systems, Ph.D. thesis, Department of Automatic Control, Lund Institute of Technology, Sweden (1999).

[46] W.-J. Wang, C.-H. Sun, Relaxed stability and stabilization conditions for a TS fuzzy discrete system, Fuzzy Sets and Systems 156 (2) (2005) 208-225.

[47] R. Marino, P. Tomei, Adaptive observers with arbitrary exponential rate of convergence for nonlinear systems, IEEE Transactions on Automatic Control 40 (7) (1995) 1300-1304.

[48] R. Marino, G. Santosuosso, P. Tomei, Robust adaptive observers for nonlinear systems with bounded disturbances, IEEE Transactions on Automatic Control 46 (6) (2001) 967-972.

[49] C.-H. Hyun, J.-H. Kim, E. Kim, M. Park, Adaptive fuzzy observer based synchronization design and secure communications of chaotic systems, Chaos, Solitons \& Fractals 27 (4) (2006) 930-940.

[50] P. M. Frank, Fault diagnosis in dynamic systems using analytical and knowledge-based redundancy: A survey and some new results, Automatica 26 (3) (1990) 459-474.

[51] X. Zhang, T. Parisini, M. M. Polycarpou, Sensor bias fault isolation in a class of nonlinear systems, IEEE Transactions on Automatic Control 50 (3) (2005) $370-376$.

[52] R. Nichols, R. Reichert, W. Rugh, Gain scheduling for $H_{\infty}$ controllers: A flight control example, IEEE Transactions on Control Systems Technology 2 (2) (1993) 69-79. 
[53] J. Löfberg, YALMIP: a toolbox for modeling and optimization in MATLAB, in: Proceedings of the CACSD Conference, Taipei, Taiwan, 2004, pp. 284-289. 